\title{
GLOBAL REGULARITY AND BOUNDS FOR SOLUTIONS OF PARABOLIC EQUATIONS FOR PROBABILITY MEASURES
}

\author{
VLADIMIR I. BOGACHEV, MICHAEL RÖCKNER, AND STANISLAV V. SHAPOSHNIKOV
}

Given a second order parabolic operator

$$
L u(t, x):=\frac{\partial u(t, x)}{\partial t}+a^{i j}(t, x) \partial_{x_{i}} \partial_{x_{j}} u(t, x)+b^{i}(t, x) \partial_{x_{i}} u(t, x),
$$

we consider the weak parabolic equation $L^{*} \mu=0$ for Borel probability measures on $(0,1) \times \mathbb{R}^{d}$. The equation is understood as the equality

$$
\int_{(0,1) \times \mathbb{R}^{d}} L u d \mu=0
$$

for all smooth functions $u$ with compact support in $(0,1) \times \mathbb{R}^{d}$. This equation is satisfied for the transition probabilities of the diffusion process associated with $L$. We show that under broad assumptions $\mu$ has the form $\mu=\varrho(t, x) d t d x$, where the function $x \mapsto \varrho(t, x)$ is Sobolev, $\left|\nabla_{x} \varrho(x, t)\right|^{2} / \varrho(t, x)$ is Lebesgue integrable over $[0, \tau] \times \mathbb{R}^{d}$, and $\varrho \in L^{p}\left([0, \tau] \times \mathbb{R}^{d}\right)$ for all $p \in$ $[1,+\infty)$ and $\tau<1$. Moreover, a sufficient condition for the uniform boundedness of $\varrho$ on $[0, \tau] \times \mathbb{R}^{d}$ is given.

Keywords: parabolic equations for measures, transition probabilities, regularity of solutions of parabolic equations, estimates of solutions of parabolic equations.

AMS Subject Classification: 35K10, 35K12, 60J35, 60J60, 47D07

\section{INTRODUCTION AND NOTATION}

The objective of this work is to give efficient conditions for the global Sobolev regularity and integrability of densities of solutions of the parabolic equations of the form

$$
L^{*} \mu=0
$$

for Borel measures $\mu$ on $(0,1) \times \mathbb{R}^{d}$. Such equations have been recently investigated in [1], 2], 3], 16]. Here $L$ is a second order parabolic operator

$$
L u(t, x):=\frac{\partial u(t, x)}{\partial t}+a^{i j}(t, x) \partial_{x_{i}} \partial_{x_{j}} u(t, x)+b^{i}(t, x) \partial_{x_{i}} u(t, x),
$$

and the interpretation of our equation is the following. We shall say that a Borel probability measure $\mu$ on $(0,1) \times \mathbb{R}^{d}$ represented in the form $\mu(d t d x)=\mu_{t}(d x) d t$ by means of a family of Borel measures $\left(\mu_{t}\right)_{t \in[0,1)}$ on $\mathbb{R}^{d}$ satisfies the weak parabolic equation (1.1) if the functions $a^{i j}$ and $b^{i}$ are integrable on every compact set in $(0,1) \times \mathbb{R}^{d}$ with respect to the measure $\mu=\mu_{t} d t$ and, for every $u \in C_{0}^{\infty}\left((0,1) \times \mathbb{R}^{d}\right)$, one has

$$
\int_{(0,1) \times \mathbb{R}^{d}} L u d \mu=\int_{0}^{1} \int_{\mathbb{R}^{d}} L u(t, x) \mu_{t}(d x) d t=0 .
$$

We shall say that $\mu$ satisfies the initial condition $\mu_{0}=\nu$ at $t=0$ if $\nu$ is a Borel measure on $\mathbb{R}^{d}$ and

$$
\lim _{t \rightarrow 0} \int_{\mathbb{R}^{d}} \zeta(x) \mu_{t}(d x)=\int_{\mathbb{R}^{d}} \zeta(x) \nu(d x)
$$

for all $\zeta \in C_{0}^{\infty}\left(\mathbb{R}^{d}\right)$.

The same definitions are introduced in the case where $\mathbb{R}^{d}$ is replaced by an open set $\Omega \subset \mathbb{R}^{d}$ or by an open set in a Riemannian manifold.

This work has been supported by the projects RFBR 04-01-00748, the Scientific Schools Grant 1758.2003.1, DFG 436 RUS 113/343/0(R), INTAS 03-51-5018, and the SFB 701 at the University of Bielefeld. 
Equation (1.1) is satisfied for the transition probabilities of the diffusion process governed by the stochastic differential equation

$$
d \xi_{t}=\sqrt{2 A\left(t, \xi_{t}\right)} d w_{t}+b\left(t, \xi_{t}\right) d t
$$

provided that such a diffusion exists and the coefficients $A$ and $b$ satisfy certain conditions. However, (1.1) can be considered regardless of any probabilistic assumptions. Moreover, a study of this equation in a purely analytical setting may be useful for constructing an associated diffusion (see [16]).

Our main result states that the density $\varrho$ of any solution has the property that $\varrho(t, \cdot)$ is Sobolev on $\mathbb{R}^{d}$ and $|\nabla \varrho(t, x)|^{2} / \varrho(t, x)$ is integrable over $[0, \tau] \times \mathbb{R}^{d}$ provided that the functions $|b|$ and $\ln (|x|+1)$ are in $L^{2}(\mu)$, the coefficient $A$ is uniformly bounded, uniformly invertible and uniformly Lipschitzian in $x$, and the initial distribution $\mu_{0}=\varrho(0, \cdot) d x$ has finite entropy. The assumptions on $A$ can be relaxed if $b$ has certain additional local integrability. An efficient condition in terms of Lyapunov functions is given in order to ensure the square integrability of $|b|$ and $\ln (|x|+1)$ with respect to the solution $\mu$. The main result enables us to show that $\varrho$ belongs to all $L^{p}\left([0, \tau] \times \mathbb{R}^{d}\right)$ whenever $\tau<1$, provided that $\sup _{t}\|b(t, \cdot)\|_{L^{d}\left(\mu_{t}\right)}<\infty$ and $\varrho(0, \cdot) \in L^{p}\left(\mathbb{R}^{d}\right)$ for all $p \geq 1$. If $|b| \in L^{\beta}(\mu)$ for some $\beta>d+2$ and $\varrho(0, \cdot) \in L^{\infty}\left(\mathbb{R}^{d}\right)$, then the density $\varrho$ is uniformly bounded on $[0, \tau] \times \mathbb{R}^{d}$ whenever $\tau<1$. By using this assertion we obtain pointwise upper bounds of the form $\varrho(t, x) \leq \Phi(x)^{-1}$. Note that unlike many known results on the global boundedness of solutions, it is not required here that the drift term be dissipative or potential.

Analogous results in the elliptic case have been obtained in [7], 4], 8], 14], 5] and [6]. One might regard the elliptic case as the situation when the solution and the coefficients are independent of time. Then our parabolic result does not recover the elliptic one, because the initial distribution (which in this case coincides with the solution) must have finite entropy, and the latter assumption cannot be completely removed in the parabolic case. On the other hand, a reasonable parabolic analogue of the elliptic result might be as follows: the integrability of $|\varrho|^{2} / \varrho$ on $\left[\tau_{1}, \tau_{2}\right] \times \mathbb{R}^{d}$ for any closed interval $\left[\tau_{1}, \tau_{2}\right] \subset$ $(0,1)$ without restrictions on the initial distribution. So far we have not succeeded in investigating this second possibility. Of course, if for some $\tau>0$ the measure $\mu_{\tau}$ has finite entropy, then our hypotheses are satisfied on $[\tau, 1]$.

Our result will be applied in a forthcoming paper on the uniqueness problem for parabolic equations for measures. It can also be useful in the study of transition probabilities of diffusion processes and in Nelson's dynamics (see [9], 10], 11], 12, [15]).

Let $W^{p, 1}\left(\mathbb{R}^{d}\right)$ denote the Sobolev space of functions that belong to $L^{p}\left(\mathbb{R}^{d}\right)$ with their generalized partial derivatives. This space is equipped with the standard norm

$$
\|f\|_{W^{p, 1}}:=\|f\|_{p}+\|\nabla f\|_{p}
$$

where $\|\cdot\|_{p}$ denotes the $L^{p}\left(\mathbb{R}^{d}\right)$-norm on scalar or vector functions. The symbol $L^{p, q}$, where $1 \leq p, q<\infty$, will stand for the space of all measurable functions $f$ on $[0,1] \times \mathbb{R}^{d}$ with finite norm

$$
\|f\|_{p, q}:=\left(\int_{0}^{1}\left(\int_{\mathbb{R}^{d}}|f(t, x)|^{p} d x\right)^{q / p} d t\right)^{1 / q} .
$$

The space $L^{p, \infty}$, where $1 \leq p<\infty$, consists of all measurable functions $f$ on $[0,1] \times \mathbb{R}^{d}$ with $t \mapsto\|f(t, \cdot)\|_{L^{p}\left(\mathbb{R}^{d}\right)} \in L^{\infty}[0,1]$. For analogous spaces of functions on $[0, \tau] \times \mathbb{R}^{d}$ we used the notation $L^{p, q}\left([0, \tau] \times \mathbb{R}^{d}\right)$. Finally, $\mathbb{H}^{p, q}\left([0, \tau] \times \mathbb{R}^{d}\right)$ denotes the space of all measurable functions $f$ on $[0, \tau] \times \mathbb{R}^{d}$ with finite norm

$$
\|f\|_{\mathbb{H}^{p, q}\left([0, \tau] \times \mathbb{R}^{d}\right)}:=\left(\int_{0}^{\tau}\|f(t, \cdot)\|_{W^{p, 1}}^{q} d t\right)^{1 / q} .
$$

For simplicity of notation the gradient of a function $u$ on $(0,1) \times \mathbb{R}^{d}$ with respect to the argument from $\mathbb{R}^{d}$ is denoted by $\nabla$ u, i.e.,

$$
\nabla u(t, x):=\nabla_{x} u(t, x)=\left(\partial_{x_{i}} u(t, x), \ldots, \partial_{x_{d}} u(t, x)\right)
$$


We use the standard rule of summation with respect to repeated indices, e.g.,

$$
\partial_{x_{i}} a^{i j}:=\sum_{i=1}^{d} \partial_{x_{i}} a^{i j}, \quad a^{i j} \partial_{x_{i}} \partial_{x_{j}} u:=\sum_{i, j=1}^{d} a^{i j} \partial_{x_{i}} \partial_{x_{j}} u .
$$

We say that a nonnegative measure $\mu_{0}$ on $\mathbb{R}^{d}$ has finite entropy if $\mu_{0}=\varrho_{0} d x$ and $\varrho_{0} \ln \varrho_{0} \in L^{1}\left(\mathbb{R}^{d}\right)$, where we set $0 \ln 0:=0$. The entropy of $\varrho_{0}$ is the integral of $\varrho_{0} \ln \varrho_{0}$.

It was shown in [3] that if the coefficient $A$ is nondegenerate, then $\mu$ is absolutely continuous with respect to Lebesgue measure on $(0,1) \times \mathbb{R}^{d}$. The corresponding density will be denoted by $\varrho$.

A sufficient condition for the existence of a solution in the class of probability measures is the following (see 2] which improves [1]). The coefficients $a^{i j}$ and $b^{i}$ are Borel functions on $[0,1] \times \mathbb{R}^{d}$ such that $A(t, x)=\left(a^{i j}(t, x)\right)$ is a nonnegative symmetric matrix and there is $p>d+2$ such that, for every ball $B$ and all $i, j \leq d$, one has

$(\mathrm{C} 1) \inf _{(t, x) \in[0,1] \times B} \operatorname{det} A(t, x) \geq M_{1}(B)>0$ and $\sup _{t \in[0,1]}\left\|a^{i j}(t, \cdot)\right\|_{W^{p, 1}(B)} \leq M_{2}(B)$,

$(\mathrm{C} 2) \sup _{t \in[0,1]}\left\|b^{i}(t, \cdot)\right\|_{L^{p}(B)} \leq M_{3}(B)$.

If $(\mathrm{C} 1)$ and $(\mathrm{C} 2)$ are fulfilled and there is a nonnegative function $V$ on $\mathbb{R}^{d}$ such that $\lim _{|x| \rightarrow+\infty} V(x)=+\infty$ and $L V \leq c_{1} V+c_{2}$ for some constants $c_{1}$ and $c_{2}$, then a solution $\mu=\mu_{t}(d x) d t$ with probability measures $\mu_{t}$ exists for every initial distribution $\mu_{0}$ and the function

$$
t \mapsto \int_{\mathbb{R}^{d}} \zeta(x) \mu_{t}(d x)
$$

is continuous on $[0,1)$ for every $\zeta \in C_{0}^{\infty}\left(\mathbb{R}^{d}\right)$. Moreover, if $V \in L^{1}\left(\mu_{0}\right)$, then

$$
\int_{0}^{1} \int_{\mathbb{R}^{d}} V(x) \mu_{t}(d x) d t<\infty .
$$

In addition, if $L V \leq c_{2}$, then

$$
\int_{\mathbb{R}^{d}} V(x) \mu_{t}(d x) \leq c_{2}
$$

for almost every $t$. For example, if the coefficient $A$ is uniformly bounded and

$$
\langle b(t, x), x\rangle \leq k_{1}|x|^{2}+k_{2},
$$

then we take the function $V(x)=\ln \left(|x|^{2}+1\right)$. This gives an estimate $L V \leq$ const, hence the integrals of $\ln (|x|+1)$ against $\mu_{t}$ are uniformly bounded provided that $\ln (|x|+1) \in$ $L^{1}\left(\mu_{0}\right)$. If the coefficient $A$ is uniformly bounded and

$$
\langle b(t, x), x\rangle \leq k_{1}|x|^{2} \ln (|x|+1)+k_{2},
$$

then we set $V(x)=\left|\ln \left(|x|^{2}+1\right)\right|^{2}$ and obtain $L V \leq c_{1} V+c_{2}$, hence $|\ln (|x|+1)|^{2}$ is $\mu$-integrable provided that it is $\mu_{0}$-integrable.

Our main estimate will be established in the two cases corresponding to two different approaches:

1) when (C1) is replaced by a stronger assumption and (C2) is replaced by the condition that $|b|, \ln \max (|x|, 1) \in L^{2}(\mu)$,

2) the condition $|b|, \ln \max (|x|, 1) \in L^{2}(\mu)$ is imposed in addition to (C1), (C2) and a certain global condition on $A$.

Set

$$
\Theta_{A}(t, x):=\sum_{j=1}^{d}\left|\sum_{i=1}^{d} \partial_{x_{i}} a^{i j}(t, x)\right|
$$




\section{BOUNDS ON LOGARITHMIC GRADIENTS}

Our first main result establishes the square integrability of the logarithmic gradient of $\mu$, i.e., the mapping $\nabla \varrho / \varrho$, with respect to $\mu$. If $\varrho(t, \cdot) \in W_{l o c}^{1,1}$, then we use the following convention: $\nabla \varrho(t, x) / \varrho(t, x):=0$ if $\varrho(t, x)=0$.

Theorem 2.1. Suppose $\mu$, where each $\mu_{t}$ is a probability measure, satisfies (1.1), (1.3). Let

(i) the mapping $A$ be uniformly bounded with $A(t, x) \geq \alpha \cdot I$ for some constant $\alpha>0$, and let the functions $x \mapsto a^{i j}(t, x)$ be Lipschitzian with constant $\lambda$,

(ii) $|b| \in L^{2}(\mu)$.

Assume also that the function $\Lambda(x):=\ln \max (|x|, 1)$ is in $L^{2}(\mu)$ (which is the case if, e.g., $\langle b(t, x), x\rangle \leq C_{1}|x|^{2} \Lambda(x)+C_{2}$ with some constants $C_{1}$ and $C_{2}$ and $\left.\Lambda \in L^{2}\left(\mu_{0}\right)\right)$. If $\mu_{0}$ has finite entropy, then $\mu_{t}=\varrho(t, \cdot) d x$, where $\varrho(t, \cdot) \in W_{\text {loc }}^{1,1}$, and for each $\tau<1$ one has

$$
\int_{0}^{\tau} \int_{\mathbb{R}^{d}} \frac{|\nabla \varrho(t, x)|^{2}}{\varrho(t, x)} d x d t<\infty .
$$

In particular, we have $\left.\sqrt{\varrho} \in \mathbb{H}^{2,2}\left([0, \tau] \times \mathbb{R}^{d}\right)\right)$ and $\left.\varrho \in L^{d /(d-2), 1}\left([0, \tau] \times \mathbb{R}^{d}\right)\right)$ if $d>2$, and $\left.\varrho \in L^{s, 1}\left([0, \tau] \times \mathbb{R}^{d}\right)\right)$ for all $s \in[1, \infty)$ if $d=2$.

If the integrals $\int_{\mathbb{R}^{d}} \varrho(t, x) \Lambda(x) d x$ are bounded as $t \rightarrow 1$ (which is the case, e.g., if $\langle b(t, x), x\rangle \leq C_{1}|x|^{2}+C_{2}$ with some constants $C_{1}$ and $C_{2}$ and $\left.\Lambda \in L^{1}\left(\mu_{0}\right)\right)$, then (2.1) is true for $\tau=1$.

Proof. We shall use the following fact (see, e.g., [4, Lemma 2.1]): given two nonnegative functions $f_{1}, f_{2} \in L^{1}\left(\mathbb{R}^{d}\right)$, for any measurable function $\psi$ such that $|\psi|^{2} f_{1} \in L^{1}\left(\mathbb{R}^{d}\right)$ one has

$$
\int_{\mathbb{R}^{d}} \frac{\left|\left(\psi f_{1}\right) * f_{2}\right|^{2}}{f_{1} * f_{2}} d x \leq \int_{\mathbb{R}^{d}}|\psi|^{2} f_{1} d x \int_{\mathbb{R}^{d}} f_{2} d x,
$$

where $\left|\left(\psi f_{1}\right) * f_{2}(x)\right|^{2} /\left(f_{1} * f_{2}(x)\right):=0$ if $f_{1} * f_{2}(x)=0$.

For a function $w \in C_{0}^{\infty}\left(\mathbb{R}^{d}\right)$ we set

$$
\varrho * w(t, x):=\int_{\mathbb{R}^{d}} w(x-y) \varrho(t, y) d y, \quad x \in \mathbb{R}^{d} .
$$

Here and in what follows the convolutions are always taken with respect to the variable from $\mathbb{R}^{d}$. Equation (1.2) and the inclusion $|b| \in L^{2}(\mu)$ yield that the following equality holds in Sobolev's sense:

$$
\partial_{t}(\varrho * w)=\left(a^{i j} \varrho\right) * \partial_{x_{i}} \partial_{x_{j}} w-\left(b^{i} \varrho\right) * \partial_{x_{i}} w .
$$

We shall deal with a version of $\varrho * w$ (denoted by the same symbol) defined by the formula

$$
\varrho * w(t, x):=\varrho * w(0, x)+\int_{0}^{t} v(s, x) d s,
$$

where $v$ is the right-hand side of (2.4). Since $|b| \in L^{2}(\mu)$ and the functions $a^{i j}$ are bounded, one has $v \in L^{1}\left([0,1] \times \mathbb{R}^{d}\right)$. Hence the function $\varrho * w$ is absolutely continuous in $t$ on $[0,1]$ and belongs to the class $C_{b}^{\infty}\left(\mathbb{R}^{d}\right)$ in $x$. For almost every $t$, the indicated version coincides for all $x$ with the initial version defined by the convolution. It will be important as well that this is true for $t=0$. Since the initial version does not exceed $\sup _{x}|w(x)|$ in the absolute value, the same is true for our new version for almost all $t$, and then pointwise by the continuity in $t$. It is readily seen from conditions (i) and (ii) that the aforementioned properties, including (2.3), also remain valid for the functions

$$
w_{\varepsilon}(x)=\varepsilon^{-d} g(x / \varepsilon)
$$


where $g$ is the standard Gaussian density and $\varepsilon \in(0,1)$. Below we take for $\varepsilon$ only numbers of the form $1 / n, n \in \mathbb{N}$. Let us set

$$
\varrho_{\varepsilon}:=\varrho * w_{\varepsilon}, \quad f_{\varepsilon}(t, x):=\varrho_{\varepsilon}(t, x)+\varepsilon \max (1,|x|)^{-d-1},
$$

where we take for $\varrho_{\varepsilon}$ the version indicated in (2.4). Since the function $\varrho \Lambda$ is integrable, one can find $\tau$ as close to 1 as we like such that

$$
\int_{\mathbb{R}^{d}} \varrho(\tau, x) \Lambda(x) d x<\infty .
$$

A number $\tau$ for which (2.5) is fulfilled can be chosen in such a way that for each $\varepsilon=1 / n$, our version of $\varrho_{\varepsilon}(\tau, x)$ will coincide with the convolution $\varrho(\tau, \cdot) * w_{\varepsilon}(x)$ for all $x$. Then the easily verified inequality $\ln \max (|x+y|, 1) \leq \ln \max (|x|, 1)+|y|$ yields

$$
\begin{aligned}
\int_{\mathbb{R}^{d}} & f_{\varepsilon}(\tau, x) \Lambda(x) d x \leq \int_{\mathbb{R}^{d}} \varrho_{\varepsilon}(\tau, x) \Lambda(x) d x+\varepsilon \int_{\mathbb{R}^{d}} \max (|x|, 1)^{-d-1} \Lambda(x) d x \\
& \leq \int_{\mathbb{R}^{d}} \varrho(\tau, x) \Lambda(x) d x+\int_{\mathbb{R}^{d}}|y| w_{\varepsilon}(y) d y+\varepsilon \int_{\mathbb{R}^{d}} \max (|x|, 1)^{-d-1} \Lambda(x) d x \leq M_{1},
\end{aligned}
$$

where $M_{1}$ is a number independent of $\varepsilon$. By (2.3) we have

$$
\int_{0}^{\tau} \int_{\mathbb{R}^{d}} \partial_{t}\left(\varrho * w_{\varepsilon}\right) \ln f_{\varepsilon} d x d t=\int_{0}^{\tau} \int_{\mathbb{R}^{d}}\left[\left(a^{i j} \varrho\right) * \partial_{x_{i}} \partial_{x_{j}} w_{\varepsilon}-\left(b^{i} \varrho\right) * \partial_{x_{i}} w_{\varepsilon}\right] \ln f_{\varepsilon} d x d t,
$$

because $\left|f_{\varepsilon}\right| \leq c_{1}+c_{2} \Lambda$ with some constants $c_{1}$ and $c_{2}$ and the functions $\left(\left(b^{i} \varrho\right) * \partial_{x_{i}} w_{\varepsilon}\right) \Lambda$ and $\left(\left(a^{i j} \varrho\right) * \partial_{x_{i}} \partial_{x_{j}} w_{\varepsilon}\right) \Lambda$ are integrable on $(0,1) \times \mathbb{R}^{d}$. Indeed, since $\varrho *\left|\partial_{x_{i}} w_{\varepsilon}\right|>0$, one has

$$
\left(\left(b^{i} \varrho\right) * \partial_{x_{i}} w_{\varepsilon}\right) \Lambda=\left(\left(b^{i} \varrho\right) * \partial_{x_{i}} w_{\varepsilon}\right)\left(\left|\partial_{x_{i}} w_{\varepsilon}\right| * \varrho\right)^{-1 / 2}\left(\left|\partial_{x_{i}} w_{\varepsilon}\right| * \varrho\right)^{1 / 2} \Lambda .
$$

Then $\left(\left(b^{i} \varrho\right) * \partial_{x_{i}} w_{\varepsilon}\right)\left(\left|\partial_{x_{i}} w_{\varepsilon}\right| * \varrho\right)^{-1 / 2} \in L^{2}\left((0,1) \times \mathbb{R}^{d}\right)$ by (2.2) and the inclusion $|b| \in L^{2}(\mu)$. In addition, $\left(\left|\partial_{x_{i}} w_{\varepsilon}\right| * \varrho\right)^{1 / 2} \Lambda \in L^{2}\left((0,1) \times \mathbb{R}^{d}\right)$ by the estimate

$$
|\ln \max (|x+y|, 1)|^{2} \leq 4+2|\ln \max (|x|, 1)|^{2}+2|\ln \max (|y|, 1)|^{2}
$$

and the same computations as in (2.6). Similarly we verify the integrability of the function $\left[\left(a^{i j} \varrho\right) * \partial_{x_{i}} \partial_{x_{j}} w_{\varepsilon}\right] \Lambda$ on $(0,1) \times \mathbb{R}^{d}$. We observe that one can integrate by parts on the right in (2.6). Indeed,

$$
\int_{0}^{\tau} \int_{\mathbb{R}^{d}} \frac{\left|\nabla f_{\varepsilon}\right|^{2}}{f_{\varepsilon}} d x d t \leq 2 \int_{0}^{\tau} \int_{\mathbb{R}^{d}} \frac{\left|\nabla \varrho_{\varepsilon}\right|^{2}}{\varrho_{\varepsilon}} d x d t+2 \varepsilon(d+1)^{2} \int_{\{|x| \geq 1\}}|x|^{-d-3} d x
$$

which is finite by (2.2) , since $\nabla \varrho_{\varepsilon}=\left(\nabla w_{\varepsilon}\right) * \varrho$ and $\left|\nabla w_{\varepsilon}\right|^{2} / w_{\varepsilon} \in L^{1}\left(\mathbb{R}^{d}\right)$. In addition, one has $\left[\left(b^{i} \varrho\right) * w_{\varepsilon}\right] \varrho_{\varepsilon}^{-1 / 2} \in L^{2}\left((0,1) \times \mathbb{R}^{d}\right)$ again by (2.2) and the inclusion $|b| \in L^{2}(\mu)$. Similarly, we have $\left[\left(a^{i j} \varrho\right) * \partial_{x_{i}} w_{\varepsilon}\right] \varrho_{\varepsilon}^{-1 / 2} \in L^{2}\left((0,1) \times \mathbb{R}^{d}\right)$. Since $f_{\varepsilon}>\varrho_{\varepsilon}$, one has

$$
\partial_{x_{i}} f_{\varepsilon}\left(\partial_{x_{j}}\left[\left(a^{i j} \varrho\right) * w_{\varepsilon}\right]-\left(b^{i} \varrho\right) * w_{\varepsilon}\right) f_{\varepsilon}^{-1} \in L^{1}\left(\mathbb{R}^{d}\right) \text {. }
$$

Therefore,

$$
\int_{0}^{\tau} \int_{\mathbb{R}^{d}} \partial_{t} \varrho_{\varepsilon} \ln f_{\varepsilon} d x d t=-\int_{0}^{\tau} \int_{\mathbb{R}^{d}} \frac{\partial_{x_{i}} f_{\varepsilon}}{f_{\varepsilon}}\left(\partial_{x_{j}}\left[\left(a^{i j} \varrho\right) * w_{\varepsilon}\right]-\left(b^{i} \varrho\right) * w_{\varepsilon}\right) d x d t .
$$

The integrand on the left can be written as $\partial_{t}\left(f_{\varepsilon} \ln f_{\varepsilon}\right)-\partial_{t} \varrho_{\varepsilon}$. Since the integrals of $\varrho_{\varepsilon}(\tau, x)$ and $\varrho_{\varepsilon}(0, x)$ in $x$ equal one, we see that the left-hand side of (2.8) equals

$$
L_{\varepsilon}:=\int_{\mathbb{R}^{d}}\left[f_{\varepsilon}(\tau, x) \ln f_{\varepsilon}(\tau, x)-f_{\varepsilon}(0, x) \ln f_{\varepsilon}(0, x)\right] d x .
$$

Since $f_{\varepsilon}(\tau, \cdot) \ln f_{\varepsilon}(\tau, \cdot) \in L^{1}\left(\mathbb{R}^{d}\right)$ by (2.6) and the estimate $\left|\ln f_{\varepsilon}\right| \leq c_{1}+c_{2} \Lambda$, one has $f_{\varepsilon}(0, \cdot) \ln f_{\varepsilon}(0, \cdot) \in L^{1}\left(\mathbb{R}^{d}\right)$. We need a lower bound on $L_{\varepsilon}$. To this end, we observe that 
by the convexity of the function $s \mapsto s \ln s$ on $(0,+\infty)$ and Jensen's inequality one has

$$
\begin{aligned}
& \int_{\mathbb{R}^{d}} f_{\varepsilon}(0, x) \ln f_{\varepsilon}(0, x) d x \\
& \leq \int_{\mathbb{R}^{d}} \varrho_{\varepsilon}(0, x) \ln \left(2 \varrho_{\varepsilon}(0, x)\right) d x+\int_{\mathbb{R}^{d}} \varepsilon \max (|x|, 1)^{-d-1} \ln \left(2 \varepsilon \max (|x|, 1)^{-d-1}\right) d x \\
& \leq \ln 2+\int_{\mathbb{R}^{d}} \varrho_{\varepsilon}(0, x) \ln \varrho_{\varepsilon}(0, x) d x+\varepsilon \ln 2 \int_{\mathbb{R}^{d}} \max (|x|, 1)^{-d-1} d x \\
& \leq \ln 2+\int_{\mathbb{R}^{d}} \varrho_{0}(x) \ln \varrho_{0}(x) d x+\varepsilon \ln 2 \int_{\mathbb{R}^{d}} \max (|x|, 1)^{-d-1} d x=: M(\varepsilon) .
\end{aligned}
$$

On the other hand, (2.6) gives

$$
\int_{\mathbb{R}^{d}} f_{\varepsilon}(\tau, x) \ln f_{\varepsilon}(\tau, x) d x \geq-(d+1) \int_{\mathbb{R}^{d}} f_{\varepsilon}(\tau, x) \Lambda(x) d x-\varepsilon M_{1}(d+1)=:-K(\varepsilon) .
$$

Note that for any bounded Borel function $a$ on $(0,1) \times \mathbb{R}^{d}$ that is Lipschitzian in the second argument with Lipschitz norm $\lambda$, for every $j$ we have

$$
\partial_{x_{j}}\left[(a \varrho) * w_{\varepsilon}\right](t, x)=a(t, x) \partial_{x_{j}} \varrho_{\varepsilon}(t, x)+\int_{\mathbb{R}^{d}} \partial_{x_{j}} w_{\varepsilon}(x-y)[a(t, y)-a(t, x)] \varrho(t, y) d y
$$

and

$$
\begin{aligned}
& \mid \int_{\mathbb{R}^{d}} \partial_{x_{j}} w_{\varepsilon}(x-y)[a(t, y)-a(t, x)] \varrho(t, y) d y\left|\leq \lambda \int_{\mathbb{R}^{d}}\right| \partial_{x_{j}} w_{\varepsilon}(x-y)|| y-x \mid \varrho(t, y) d y \\
& \leq \lambda \int_{\mathbb{R}^{d}} \varepsilon^{-d} \frac{|x-y|^{2}}{\varepsilon^{2}} g\left(\frac{x-y}{\varepsilon}\right) \varrho(t, y) d y=\lambda\left(\varrho * q_{\varepsilon}\right)(t, x), \quad(2
\end{aligned}
$$

where $q_{\varepsilon}(x):=w_{\varepsilon}(x)|x / \varepsilon|^{2}, x \in \mathbb{R}^{d}$. Let us note for the sequel that in the derivation of (2.8) and (2.9) we have not used the $\mu$-integrability of $\Lambda^{2}$ and the existence of entropy of $\mu_{0}$. By using (2.8) and (2.9) we obtain

$$
\begin{aligned}
\int_{0}^{1} \int_{\mathbb{R}^{d}} a^{i j} \frac{\partial_{x_{i}} f_{\varepsilon}}{f_{\varepsilon}} \partial_{x_{j}} f_{\varepsilon} d x d t=\int_{0}^{1} \int_{\mathbb{R}^{d}} \frac{\partial_{x_{i}} f_{\varepsilon}}{f_{\varepsilon}}\left[\left(b^{i} \varrho\right) * w_{\varepsilon}+\varepsilon a^{i j} \partial_{x_{j}} \frac{1}{\max (|x|, 1)^{d+1}}\right] d x d t \\
-\int_{0}^{1} \int_{\mathbb{R}^{d}}\left(\frac{\partial_{x_{i}} f_{\varepsilon}(t, x)}{f_{\varepsilon}(t, x)} \int_{\mathbb{R}^{d}} \partial_{x_{j}} w_{\varepsilon}(x-y)\left[a^{i j}(t, y)-a^{i j}(t, x)\right] \varrho(t, y) d y\right) d x d t-L_{\varepsilon} .
\end{aligned}
$$

The right-hand side of this equality does not exceed

$$
\begin{aligned}
\left(\int_{0}^{\tau} \int_{\mathbb{R}^{d}} \frac{\left|\nabla f_{\varepsilon}\right|^{2}}{f_{\varepsilon}} d x d t\right)^{1 / 2}\left[\left(\int_{0}^{\tau} \int_{\mathbb{R}^{d}} \frac{\sum_{i=1}^{d}\left[\left(b^{i} \varrho\right) * w_{\varepsilon}\right]^{2}}{f_{\varepsilon}} d x d t\right)^{1 / 2}+\varepsilon M C_{d}\right] \\
+d^{3 / 2} \lambda\left(\int_{0}^{\tau} \int_{\mathbb{R}^{d}} \frac{\left|\nabla f_{\varepsilon}\right|^{2}}{f_{\varepsilon}} d x d t\right)^{1 / 2}\left(\int_{0}^{\tau} \int_{\mathbb{R}^{d}} \frac{\left(\varrho * q_{\varepsilon}\right)^{2}}{f_{\varepsilon}} d x d t\right)^{1 / 2}+M(\varepsilon)+K(\varepsilon),
\end{aligned}
$$

where $M=\sup _{t, x}\|A(t, x)\|$ and $C_{d}$ is the integral of $(d+1)^{2}|x|^{-d-3}$ over the set $\{|x| \geq 1\}$. By (2.2) we have

$$
\begin{gathered}
\int_{0}^{\tau} \int_{\mathbb{R}^{d}} \frac{\left[\left(b^{i} \varrho\right) * w_{\varepsilon}\right]^{2}}{f_{\varepsilon}} d x d t \leq \int_{0}^{\tau} \int_{\mathbb{R}^{d}}\left|b^{i}\right|^{2} d \mu, \quad 1 \leq i \leq d \\
\int_{0}^{\tau} \int_{\mathbb{R}^{d}} \frac{\left(\varrho * q_{\varepsilon}\right)^{2}}{f_{\varepsilon}} d x d t \leq \gamma:=\int_{\mathbb{R}^{d}}|x|^{4} g(x) d x
\end{gathered}
$$


Since $A \geq \alpha \cdot I$, we arrive at the estimate

$$
\begin{aligned}
\alpha \int_{0}^{\tau} \int_{\mathbb{R}^{d}} \frac{\left|\nabla f_{\varepsilon}\right|^{2}}{f_{\varepsilon}} d x d t & \\
& \leq\left(\int_{0}^{\tau} \int_{\mathbb{R}^{d}} \frac{\left|\nabla f_{\varepsilon}\right|^{2}}{f_{\varepsilon}} d x d t\right)^{1 / 2}\left(\|b\|_{2, \mu}+\varepsilon M C_{d}+\lambda d^{3 / 2} \sqrt{\gamma}\right)+M(\varepsilon)+K(\varepsilon),
\end{aligned}
$$

which by the inequality $c \sqrt{x} \leq \alpha x / 2+c^{2} /(2 \alpha)$ yields the estimate

$$
\int_{0}^{\tau} \int_{\mathbb{R}^{d}} \frac{\left|\nabla f_{\varepsilon}\right|^{2}}{f_{\varepsilon}} d x d t \leq \alpha^{-2}\left(\|b\|_{2, \mu}+\varepsilon M C_{d}+\lambda d^{3 / 2} \sqrt{\gamma}\right)^{2}+2 \alpha^{-1}(M(\varepsilon)+K(\varepsilon)) .
$$

The quantities $M(\varepsilon)$ and $K(\varepsilon)$ are uniformly bounded in $\varepsilon$. Letting $\varepsilon \rightarrow 0$ we obtain that $\sqrt{\varrho(t, \cdot)} \in W^{2,1}\left(\mathbb{R}^{d}\right)$ for almost all $t \in(0,1)$. Hence $\varrho(t, \cdot) \in W^{1,1}\left(\mathbb{R}^{d}\right)$ for almost all $t \in(0, \tau)$. In addition, the integral of $|\nabla \varrho|^{2} / \varrho$ does not exceed the right-hand side of (2.11) with $\varepsilon=0$. Thus, $\left.\sqrt{\varrho} \in \mathbb{H}^{2,2}\left([0, \tau] \times \mathbb{R}^{d}\right)\right)$. By the Sobolev embedding theorem we have $\left.\varrho \in L^{d /(d-2), 1}\left([0, \tau] \times \mathbb{R}^{d}\right)\right)$ if $d>2$, and $\left.\varrho \in L^{s, 1}\left([0, \tau] \times \mathbb{R}^{d}\right)\right)$ for all $s \in[1, \infty)$ if $d=2$.

The last claim of the theorem is clear from our reasoning.

The proof yields a useful estimate

$$
\begin{aligned}
& \int_{0}^{\tau} \int_{\mathbb{R}^{d}} \frac{|\nabla \varrho|^{2}}{\varrho} d x d t \leq \alpha^{-2}\left(\|b\|_{2, \mu}+\lambda d^{3 / 2} \sqrt{\gamma}\right)^{2}+2 \ln 2 \alpha^{-1}+2 \alpha^{-1} \int_{\mathbb{R}^{d}} \varrho_{0}(x) \ln \varrho_{0}(x) d x \\
& +2 \alpha^{-1}(d+1) \int_{\mathbb{R}^{d}} \varrho(\tau, x) \Lambda(x) d x .
\end{aligned}
$$

Remark 2.1. It is clear from the proof that the entropy of $\varrho_{\varepsilon}(0, x)$ has to be estimated only from above, so in place of the integrability of $\varrho(0, x) \ln \varrho(0, x)$ it suffices to require only the integrability of $\varrho(0, x) \max (0, \ln \varrho(0, x))$ (then Jensen's inequality must be applied to the function $s \max (0, \ln s))$. This leads to the effect that in estimate (2.12) in place of $\varrho(0, x) \ln \varrho(0, x)$ we obtain $\varrho(0, x) \max (0, \ln \varrho(0, x))$. However, the obtained estimates and (2.8) Show that if we keep all other assumptions, the entropy of $\varrho(0, x)$ is finite anyway. But if no $\mu$-integrability of $\Lambda$ is required, then the situation may change. For example, if $d=1, b=0$ and $a=1 / 2$, then for any initial distribution $\mu_{0}$, the solution is given by the convolution $\mu_{0} * g_{t}$, where $g_{t}(x)=(2 \pi t)^{-1 / 2} \exp \left(-x^{2} /(2 t)\right)$. If $\mu_{0}$ has a density $\varrho_{0}$ such that $\left|\varrho_{0}^{\prime}\right|^{2} / \varrho_{0} \in L^{1}\left(\mathbb{R}^{1}\right)$, but the function $\varrho_{0} \ln \varrho_{0}$ is not integrable, then the solution $\varrho(t, x)$ has no entropy for any $t$, although the quantities $\int\left|\partial_{x} \varrho(t, x)\right|^{2} \varrho(t, x)^{-1} d x$ are uniformly bounded. The same example shows that for validity of estimate (2.1) certain conditions on the initial distribution are necessary. It suffices to take for $\mu_{0}$ Dirac's measure at the origin. Then the function $\left|\partial_{x} \varrho\right|^{2} / \varrho$ is not integrable on $(0,1) \times \mathbb{R}^{1}$. It would be interesting to find a sufficient condition on $A$ and $b$ ensuring finite entropy of $\varrho(t, \cdot)$ for $t>0$ and any initial distribution.

In Example 3.1 below and in [1 one can find conditions on the coefficients $A$ and $b$ that ensure the inclusion $|b| \in L^{2}(\mu)$.

Estimate (2.12) can be improved under additional hypotheses on $A$ and $b$.

Set $b_{0}:=\left(b_{0}^{j}\right), b_{0}^{j}=b^{j}-\partial_{x_{i}} a^{i j}$.

Theorem 2.2. Suppose $\mu$ satisfies (1.1), (1.3), where $\nu=\varrho_{0} d x, \varrho_{0}$ has finite entropy and is locally Hölder continuous. Let $A$ and $b$ satisfy $(\mathrm{C} 1)$ and $(\mathrm{C} 2)$ with some $p>d+2$. Suppose that $\left|A^{-1 / 2} b_{0}\right| \in L^{2}(\mu), \ln (1+|x|) \in L^{4}(\mu)$ and that

$$
\liminf _{r \rightarrow \infty} \int_{0}^{1} \int_{r \leq|x| \leq 2 r}\left[r^{-4}\|A(t, x)\|^{2}+r^{-2} \Theta_{A}(t, x)^{2}\right] \mu_{t}(d x) d t=0
$$


Then $\varrho(t, \cdot) \in W_{l o c}^{p, 1}$ and for almost all $\tau \in[0,1]$ one has

$\int_{0}^{\tau} \int_{\mathbb{R}^{d}}\left|\frac{\sqrt{A} \nabla \varrho}{\varrho}\right|^{2} d \mu \leq \int_{0}^{\tau} \int_{\mathbb{R}^{d}}\left|A^{-1 / 2} b_{0}\right|^{2} d \mu+2 \int_{\mathbb{R}^{d}}[\varrho(0, x) \ln \varrho(0, x)-\varrho(\tau, x) \ln \varrho(\tau, x)] d x$

and the right-hand side is finite. Under the additional assumption that $A \geq \alpha \cdot I$ for some $\alpha>0$, one has $\left.\left.\sqrt{\varrho} \in \mathbb{H}^{2,2}\left([0,1] \times \mathbb{R}^{d}\right)\right), \varrho \in L^{d /(d-2), 1}\left([0,1] \times \mathbb{R}^{d}\right)\right)$ if $d>2$ and $\left.\varrho \in L^{s, 1}\left([0,1] \times \mathbb{R}^{d}\right)\right)$ for all $s \in[1, \infty)$ if $d=2$.

Proof. By the local theory [3], we know that $\mu$ has a continuous positive density $\varrho$ such that for every ball $B$ and every closed interval $\left[t_{1}, t_{2}\right]$ in $(0,1)$ we have $\|\varrho(t, \cdot)\|_{W^{p, 1}(B)} \in$ $L^{p}\left[t_{1}, t_{2}\right]$. Let $B_{j}$ denote the closed ball of radius $j$ centered at the origin. We fix a function $\zeta \in C_{0}^{\infty}\left(\mathbb{R}^{d}\right)$ such that $\zeta(x)=1$ if $|x| \leq 1, \zeta(x)=0$ if $|x|>2$. Set $\zeta_{j}(x):=\zeta(x / j)$. For small $\varepsilon>0$ and large $k>0$, let

$$
\varrho_{k, \varepsilon}:=\min \left(k, \varrho_{\varepsilon}\right), \quad \varrho_{k}=\min (k, \varrho), \quad \Omega_{k, \varepsilon}:=\left\{\varrho_{\varepsilon}<k\right\}, \quad \Omega_{k}:=\{\varrho<k\} .
$$

As in Theorem [2.1, for almost all $\tau$ one has (2.5), which gives the integrability of $\varrho(\tau, \cdot) \ln \varrho(\tau, \cdot)$ on $\mathbb{R}^{d}$. For any $\delta>0$ and $\tau=1-\delta$ we have the equality

$$
\begin{aligned}
& \int_{\delta}^{\tau} \int_{\mathbb{R}^{d}}\left(\partial_{t} \varrho_{\varepsilon}\right)\left(\ln \varrho_{k, \varepsilon}\right) \zeta_{j}^{2} d x d t \\
= & -\int_{\delta}^{\tau} \int_{\mathbb{R}^{d}}\left(a^{i k} \partial_{x_{i}} \varrho\right) * w_{\varepsilon} \frac{\partial_{x_{k}} \varrho_{\varepsilon}}{\varrho_{\varepsilon}} \zeta_{j}^{2} I_{\Omega_{k, \varepsilon}} d x d t-2 \int_{\delta}^{\tau} \int_{\mathbb{R}^{d}}\left(a^{i k} \partial_{x_{i}} \varrho\right) * w_{\varepsilon} \partial_{x_{k}} \zeta_{j}\left(\ln \varrho_{k, \varepsilon}\right) \zeta_{j} d x d t \\
& \left.+\int_{\delta}^{\tau} \int_{\mathbb{R}^{d}} I_{\Omega_{k, \varepsilon}}\left(\left(b_{0} \varrho\right) * w_{\varepsilon}, \frac{\nabla \varrho_{\varepsilon}}{\varrho_{\varepsilon}}\right) \zeta_{j}^{2} d x d t+2 \int_{\delta}^{\tau} \int_{\mathbb{R}^{d}}\left(\left(b_{0} \varrho\right) * w_{\varepsilon}, \nabla \zeta_{j}\right)\right) \zeta_{j} \ln \varrho_{k, \varepsilon} d x d t .
\end{aligned}
$$

Since $\varrho_{\varepsilon} \partial_{t} \ln \varrho_{k, \varepsilon}=\partial_{t} \varrho_{k, \varepsilon}$, the left-hand side equals

$$
\begin{aligned}
E(j, k, \varepsilon, \delta):=\int_{\mathbb{R}^{d}} \zeta_{j}^{2}(x) \varrho_{\varepsilon}(\tau, x) \ln \varrho_{k, \varepsilon}(\tau, x) d x-\int_{\mathbb{R}^{d}} \zeta_{j}^{2}(x) \varrho_{\varepsilon}(\delta, x) \ln \varrho_{k, \varepsilon}(\delta, x) d x \\
+\int_{\mathbb{R}^{d}} \zeta_{j}^{2}(x) \varrho_{k, \varepsilon}(\delta, x) d x-\int_{\mathbb{R}^{d}} \zeta_{j}^{2}(x) \varrho_{k, \varepsilon}(\tau, x) d x
\end{aligned}
$$

Keeping $\delta>0$ fixed, letting $\varepsilon \rightarrow 0$ and using the integrability of the function $t \mapsto$ $\|\varrho(t, \cdot)\|_{W^{p, 1}\left(B_{2 j}\right)}$ on $[\delta, \tau]$ as well as the continuity and strict positivity of $\varrho$ on $[\delta, \tau] \times B_{2 j}$, we obtain

$$
\begin{aligned}
& S_{j, k, \delta}:= \int_{\delta}^{\tau} \int_{\mathbb{R}^{d}}\left(A \nabla \varrho, \frac{\nabla \varrho}{\varrho}\right) \zeta_{j}^{2} I_{\Omega_{k}} d x d t \\
&=-2 \int_{\delta}^{\tau} \int_{\mathbb{R}^{d}}\left(A \nabla \varrho, \nabla \zeta_{j}\right) \zeta_{j} \ln \varrho_{k} d x d t+\int_{\delta}^{\tau} \int_{\mathbb{R}^{d}} I_{\Omega_{k}}\left(b_{0}, \frac{\nabla \varrho}{\varrho}\right) \zeta_{j}^{2} \varrho d x d t \\
& \quad+2 \int_{\delta}^{\tau} \int_{\mathbb{R}^{d}}\left(b_{0}, \nabla \zeta_{j}\right) \zeta_{j}(\ln \varrho k) \varrho d x d t-E(j, k, \delta),
\end{aligned}
$$

where

$$
\begin{aligned}
E(j, k, \delta):=\int_{\mathbb{R}^{d}} \zeta_{j}^{2}(x) \varrho(\tau, x) \ln \varrho_{k}(\tau, x) d x & -\int_{\mathbb{R}^{d}} \zeta_{j}^{2}(x) \varrho(\delta, x) \ln \varrho_{k}(\delta, x) d x \\
& +\int_{\mathbb{R}^{d}} \zeta_{j}^{2}(x) \varrho_{k}(\delta, x) d x-\int_{\mathbb{R}^{d}} \zeta_{j}^{2}(x) \varrho_{k}(\tau, x) d x
\end{aligned}
$$


Integrating by parts in the integral of $\left(A \nabla \varrho, \nabla \zeta_{j}\right) \ln \varrho_{k} \zeta_{j}=\left(\nabla \varrho, \zeta_{j} A \nabla \zeta_{j}\right) \ln \varrho_{k}$ and writing $\left(b_{0}, \nabla \varrho\right)=\left(A^{-1 / 2} b_{0}, A^{1 / 2} \nabla \varrho\right)$, we find

$$
\begin{aligned}
S_{j, k, \delta} & =2 \int_{\delta}^{\tau} \int_{\Omega_{k}}\left(\frac{\nabla \varrho}{\varrho}, A \nabla \zeta_{j}\right) \zeta_{j} \varrho d x d t+2 \int_{\delta}^{\tau} \int_{\mathbb{R}^{d}} \operatorname{div}\left(\zeta_{j} A \nabla \zeta_{j}\right)\left(\ln \varrho_{k}\right) \varrho d x d t \\
& +\int_{\delta}^{\tau} \int_{\Omega_{k}}\left(b_{0}, \frac{\nabla \varrho}{\varrho}\right) \zeta_{j}^{2} \varrho d x d t+2 \int_{\delta}^{\tau} \int_{\mathbb{R}^{d}}\left(b_{0}, \nabla \zeta_{j}\right) \zeta_{j}\left(\ln \varrho_{k}\right) \varrho d x d t-E(j, k, \delta) \\
& \leq \sqrt{S_{j, k, \delta}}\left(2\left\|I_{\Omega_{k}} \sqrt{A} \nabla \zeta_{j}\right\|_{L^{2}(\mu)}+\left\|A^{-1 / 2} b_{0}\right\|_{L^{2}(\mu)}\right)+R_{j, k, \delta}-E(j, k, \delta)
\end{aligned}
$$

where

$$
R_{j, k, \delta}:=2 \int_{\delta}^{\tau} \int_{\mathbb{R}^{d}} \operatorname{div}\left(\zeta_{j} A \nabla \zeta_{j}\right) \ln \varrho_{k} \varrho d x d t+2 \int_{\delta}^{\tau} \int_{\mathbb{R}^{d}}\left(b_{0}, \nabla \zeta_{j}\right) \zeta_{j}\left(\ln \varrho_{k}\right) \varrho d x d t .
$$

Since $\varrho_{0}$ is Hölder continuous on $B_{2 j}$, one has $\lim _{\delta \rightarrow 0} \varrho(\delta, x)=\varrho(0, x)$ uniformly on $B_{2 j}$ (see, e.g., 13, Ch. III, Theorem 7.1 and Theorem 10.1]). Hence

$$
\lim _{\delta \rightarrow 0} E(j, k, \delta)=E(j, k, 0) .
$$

Therefore, (2.14) holds for $\delta=0$. Keeping $k$ fixed, we observe that, given $\varepsilon>0$, for all sufficiently large numbers $j$ of the form $j=r_{l}$ with $r_{l} \rightarrow \infty$ chosen according to (2.13), the quantity $R_{j, k, \delta}$ can be made smaller than $\varepsilon$ in absolute value. Indeed, it follows by the hypotheses and the estimates

$$
\sup _{x}\left|\nabla \zeta_{j}(x)\right| \leq j^{-1} \sup _{x}|\nabla \zeta(x)|, \quad \sup _{x}\left|\partial_{x_{i}} \partial_{x_{m}} \zeta_{j}(x)\right| \leq j^{-2} \sup _{x}\left|\partial_{x_{i}} \partial_{x_{m}} \zeta(x)\right|
$$

that for all $j=r_{l}$ the first term in the expression for $R_{j, k, \delta}$ can be estimated by

$$
\begin{aligned}
M\left\|\ln \varrho_{k}\right\|_{L^{2}(\mu)} r_{l}^{-2}\left(\int_{0}^{1} \int_{\left\{r_{l} \leq|x| \leq 2 r_{l}\right\}}\|A(t, x)\|^{2} \mu_{t}(d x) d t\right)^{1 / 2} & \\
& +M\left\|\ln \varrho_{k}\right\|_{L^{2}(\mu)} r_{l}^{-1}\left(\int_{0}^{1} \int_{\left\{r_{l} \leq|x| \leq 2 r_{l}\right\}} \Theta_{A}(t, x)^{2} \mu_{t}(d x) d t\right)^{1 / 2},
\end{aligned}
$$

where $M$ is a constant that depends on the maxima of the first and second derivatives of $\zeta$. The fact that $\ln \varrho_{k} \in L^{2}(\mu)$ follows by the $\mu$-integrability of $|\ln (|x|+1)|^{2}$, because on the set $\{x: \varrho(t, x) \leq 1\}$ we have $|\ln \varrho(t, x)|^{2} \sqrt{\varrho(t, x)} \leq C$, hence

$$
|\ln \varrho(t, x)|^{2} \varrho(t, x) \leq(2 d+2)^{2}(\ln (|x|+1))^{2} \varrho(t, x)+C(|x|+1)^{-d-1} .
$$

Similarly, by the Cauchy inequality and the estimate

$$
|b(t, x)| \leq\left\|A^{1 / 2}(t, x)\right\|\left|A^{-1 / 2}(t, x) b(t, x)\right|,
$$

the second term in the expression for $R_{j, k, \delta}$ is majorized by

$$
M r_{l}^{-1}\left\|A^{-1 / 2} b\right\|_{L^{2}(\mu)}\left\|\ln \varrho_{k}\right\|_{L^{4}(\mu)}^{2}\left(\int_{0}^{1} \int_{r_{l} \leq|x| \leq 2 r_{l}}\|A(t, x)\|^{2} \mu_{t}(d x) d t\right)^{1 / 2} .
$$

The quantities $E(j, k, 0)$ are bounded from below by a constant independent of $j$ and $k$, because we consider only those $\tau$ for which $\varrho(\tau, \cdot) \ln \varrho(\tau, \cdot) \in L^{1}\left(\mathbb{R}^{d}\right)$, and we have $\varrho(0, \cdot) \ln \varrho(0, \cdot) \in L^{1}\left(\mathbb{R}^{d}\right)$ by assumption. This yields that the integrals of $|\sqrt{A} \nabla \varrho / \varrho|^{2}$ over the sets $\Omega_{k}$ against $\mu$ are uniformly bounded. Letting $k \rightarrow \infty$ and then $j \rightarrow \infty$ we see that the function $|\sqrt{A} \nabla \varrho|^{2} \varrho^{-1}$ is integrable on $[0, \tau] \times \mathbb{R}^{d}$. In addition, by (2.14) its integral $S$ satisfies the inequality $S \leq \sqrt{S}\left\|A^{-1 / 2} b_{0}\right\|_{L^{2}(\mu)}+E$, where $E$ is the difference of entropies of $\varrho(0, \cdot)$ and $\varrho(\tau, \cdot)$. This yields the desired bound.

Remark 2.2. If $A$ is uniformly bounded, then the assumption $\Lambda \in L^{4}(\mu)$ in the second theorem can be relaxed to $\Lambda \in L^{2}(\mu)$. 


\section{HigheR INTEGRABILITY AND BOUNDEDNESS OF DENSITIES}

The results of the previous section show that the solutions are globally integrable in some power greater than 1 . Here we derive yet stronger integrability properties and the global boundedness under additional assumptions on the coefficients. In what follows we assume that the measure $\mu$ is given by a density $\varrho$ such that for every $t \in[0,1)$, the function $x \mapsto \varrho(t, x)$ is a probability density with respect to Lebesgue measure.

Set

$$
\|u\|_{p, q, \tau}=\left\|u I_{[0, \tau]}\right\|_{p, q}
$$

where $t \rightarrow I_{[0, \tau]}(t)$ is the indicator function of the interval $[0, \tau]$.

Lemma 3.1. Let $d>2$. For every function $u \in \mathbb{H}^{2,2}\left([0, \tau] \times \mathbb{R}^{d}\right) \bigcap L^{2, \infty}\left([0, \tau] \times \mathbb{R}^{d}\right)$, one has the inequality

$$
\|u\|_{p, q, \tau} \leq c(d, p)\left(\|\nabla u\|_{L^{2}\left([0, \tau] \times \mathbb{R}^{d}\right)}+\|u\|_{2, \infty, \tau}\right),
$$

where $2 \leq q, 2<p \leq 2 d /(d-2)$ and $1 / q+d /(2 p)=d / 4$.

Proof. Let $\delta=d / 2-d / p=2 / q$. Then

$$
\frac{(d-2) \delta}{2 d}+\frac{1-\delta}{2}=\frac{1}{p}
$$

Let $r=2 d /(p \delta(d-2))$. Then $r \geq 1(r>1$ if $p<2 d /(d-2))$ and $r^{\prime}=r /(r-1)=$ $2 /(p(1-\delta))$. Writing $|u|^{p}=|u|^{p \delta}|u|^{p(1-\delta)}$ and applying Hölder's inequality with $r$ and $r^{\prime}$ we obtain

$$
\left(\int_{\mathbb{R}^{d}}|u|^{p} d x\right)^{1 / p} \leq\left(\int_{\mathbb{R}^{d}}|u|^{2 d /(d-2)} d x\right)^{(d-2) \delta / d}\left(\int_{\mathbb{R}^{d}}|u|^{2} d x\right)^{(1-\delta) / 2} .
$$

The Sobolev inequality yields

$$
\|u(t, \cdot)\|_{p} \leq c(d, p)\|\nabla u(t, \cdot)\|_{2}^{\delta}\|u(t, \cdot)\|_{2}^{1-\delta} .
$$

Therefore, for almost all $t \in[0, \tau]$ we have

$$
\|u(t, \cdot)\|_{p} \leq c(d, p)\|\nabla u(t, \cdot)\|_{2}^{\delta}\|u\|_{2, \infty, \tau}^{1-\delta} .
$$

Since $\delta=2 / q$, one has

$$
\left(\int_{0}^{\tau}\|u(t, \cdot)\|_{p}^{q} d t\right)^{1 / q} \leq c(d, p)\left(\int_{0}^{\tau}\|\nabla u(t, \cdot)\|_{2}^{2} d t\right)^{1 / q}\left(\|u\|_{2, \infty, \tau}\right)^{1-2 / q} .
$$

By the Young inequality we obtain the required estimate.

Lemma 3.2. Suppose that hypothesis (i) of Theorem 2.1 is fulfilled and we have additionally

$$
\sup _{t \in[0,1]}\|b(t, \cdot)\|_{L^{s}\left(\mu_{t}\right)}<\infty, \quad \varrho \in L^{k s /(s-2)+1, k+(s-2) / s}\left([0, T] \times \mathbb{R}^{d}\right)
$$

with some $T \in[0,1], s>2, k \geq 2 / s$. Furthermore, let $\mu_{0}=\varrho(0, \cdot) d x$, where $\varrho(0, \cdot) \in$ $L^{k+1}\left(\mathbb{R}^{d}\right)$. Then for almost all $\tau \in[0, T]$ we have $\varrho(\tau, \cdot) \in W_{\text {loc }}^{1,1}$ and

$$
\begin{aligned}
& \frac{2}{\alpha k(k+1)} \int_{\mathbb{R}^{d}} \varrho(\tau, x)^{k+1} d x+\int_{0}^{\tau} \int_{\mathbb{R}^{d}}|\nabla \varrho(t, x)|^{2} \varrho(t, x)^{k-1} d x d t \\
& \leq C(\alpha, \lambda, d, s) \int_{0}^{\tau}\left(\int_{\mathbb{R}^{d}} \varrho(t, x)^{k s /(s-2)+1} d x\right)^{(s-2) / s} d t+\frac{2}{\alpha k(k+1)} \int_{\mathbb{R}^{d}} \varrho(0, x)^{k+1} d x,
\end{aligned}
$$

where $\alpha$ is the constant from the condition $A(t, x) \geq \alpha \cdot I$ and $C(\alpha, \lambda, d, s)$ is some number that depends only on $\alpha, \lambda, d, s$.

If in place of (3.1) we have the condition

$$
|b| \in L^{s}(\mu), \quad \varrho \in L^{k s /(s-2)+1}\left([0, T] \times \mathbb{R}^{d}\right)
$$


where $s>2, k>0$, then for almost all $\tau \in[0, T]$, one has the inequality

$$
\begin{aligned}
& \frac{2}{\alpha k(k+1)} \int_{\mathbb{R}^{d}} \varrho(\tau, x)^{k+1} d x+\int_{0}^{\tau} \int_{\mathbb{R}^{d}}|\nabla \varrho(t, x)|^{2} \varrho(t, x)^{k-1} d x d t \\
\leq & C(\alpha, \lambda, d, s)\left(\int_{0}^{\tau} \int_{\mathbb{R}^{d}} \varrho(t, x)^{k s /(s-2)+1} d x d t\right)^{(s-2) / s}+\frac{2}{\alpha k(k+1)} \int_{\mathbb{R}^{d}} \varrho(0, x)^{k+1} d x .
\end{aligned}
$$

Proof. Let $\varrho_{\varepsilon}$ be the same as in Theorem 2.1, in particular, $\varepsilon \in\{1 / n\}$. By (2.3) we have

$$
\int_{0}^{\tau} \int_{\mathbb{R}^{d}} \partial_{t}\left(\varrho * w_{\varepsilon}\right) \varphi d x d t=\int_{0}^{\tau} \int_{\mathbb{R}^{d}}\left[\left(a^{i j} \varrho\right) * \partial_{x_{i}} \partial_{x_{j}} w_{\varepsilon}-\left(b^{i} \varrho\right) * \partial_{x_{i}} w_{\varepsilon}\right] \varphi d x d t
$$

for every bounded measurable function $\varphi$ on $[0,1] \times \mathbb{R}^{d}$, because the indicated convolutions are integrable. Let us take

$$
\varphi(t, x):=\varrho_{\varepsilon}(t, x)^{k}
$$

We observe that

$$
\left[\left(a^{i j} \varrho\right) * \partial_{x_{j}} w_{\varepsilon}-\left(b^{i} \varrho\right) * w_{\varepsilon}\right] \varrho_{\varepsilon}^{k-1} \partial_{x_{i}} \varrho_{\varepsilon} \in L^{1}\left([0,1] \times \mathbb{R}^{d}\right) .
$$

Indeed, the function $\varrho_{\varepsilon}^{k}$ is bounded and the functions

$$
\left[\left(a^{i j} \varrho\right) * \partial_{x_{j}} w_{\varepsilon}-\left(b^{i} \varrho\right) * w_{\varepsilon}\right] \varrho_{\varepsilon}^{-1 / 2}, \quad\left|\nabla \varrho_{\varepsilon}\right| \varrho_{\varepsilon}^{-1 / 2}
$$

belong to $L^{2}\left([0,1] \times \mathbb{R}^{d}\right)$, as already noted in the proof of Theorem 2.1. Therefore, we can integrate by parts, which gives

$$
\int_{0}^{\tau} \int_{\mathbb{R}^{d}} \partial_{t}\left(\varrho * w_{\varepsilon}\right) \varrho_{\varepsilon}^{k} d x d t=-\int_{0}^{\tau} \int_{\mathbb{R}^{d}}\left[\left(a^{i j} \varrho\right) * \partial_{x_{j}} w_{\varepsilon}-\left(b^{i} \varrho\right) * w_{\varepsilon}\right] k \varrho_{\varepsilon}^{k-1} \partial_{x_{i}} \varrho_{\varepsilon} d x d t
$$

Let $L_{\varepsilon}$ denote the left-hand side of this equality. Then

$$
L_{\varepsilon}=\frac{1}{k+1} \int_{\mathbb{R}^{d}} \varrho_{\varepsilon}(\tau, x)^{k+1} d x-\frac{1}{k+1} \int_{\mathbb{R}^{d}} \varrho_{\varepsilon}(0, x)^{k+1} d x .
$$

Note that the integrability of $\varrho_{\varepsilon}(\tau, x)^{k+1}$ in $x$ follows by the boundedness of this function and its integrability for $k=0$. By using Hölder's inequality, we estimate $L_{\varepsilon}$ from below as follows:

$$
L_{\varepsilon} \geq \frac{1}{k+1} \int_{\mathbb{R}^{d}} \varrho_{\varepsilon}(\tau, x)^{k+1} d x-\frac{1}{k+1} \int_{\mathbb{R}^{d}} \varrho(0, x)^{k+1} d x .
$$

Let us consider the right-hand side $R_{\varepsilon}$ of equality (3.5). By using equality (2.9), we obtain

$$
\begin{gathered}
R_{\varepsilon}=-k \int_{0}^{\tau} \int_{\mathbb{R}^{d}} a^{i j} \partial_{x_{j}} \varrho_{\varepsilon} \partial_{x_{i}} \varrho_{\varepsilon} \varrho_{\varepsilon}^{k-1} d x d t \\
-k \int_{0}^{\tau} \int_{\mathbb{R}^{d}}\left(\partial_{x_{i}} \varrho_{\varepsilon}(t, x) \varrho_{\varepsilon}(t, x)^{k-1} \int_{\mathbb{R}^{d}} \partial_{x_{j}} w_{\varepsilon}(x-y)\left[a^{i j}(t, y)-a^{i j}(t, x)\right] \varrho(t, y) d y\right) d x d t \\
+k \int_{0}^{\tau} \int_{\mathbb{R}^{d}} \varrho_{\varepsilon} \partial_{x_{i}} \varrho_{\varepsilon} \varrho_{\varepsilon}^{k-1}\left[\left(b^{i} \varrho\right) * w_{\varepsilon}\right] d x d t .
\end{gathered}
$$

Hence, by (2.10) (we recall that in the derivation of (2.10) we have not used the $\mu$ integrability of $|\ln (1+|x|)|^{2}$ and the existence of entropy of $\left.\mu_{0}\right)$ we have

$$
\begin{aligned}
R_{\varepsilon} \leq- & k \alpha \int_{0}^{\tau} \int_{\mathbb{R}^{d}}\left|\nabla \varrho_{\varepsilon}\right|^{2} \varrho_{\varepsilon}^{k-1} d x d t \\
& +k d^{3 / 2} \lambda\left(\int_{0}^{\tau} \int_{\mathbb{R}^{d}}\left|\nabla \varrho_{\varepsilon}\right|^{2} \varrho_{\varepsilon}^{k-1} d x d t\right)^{1 / 2}\left(\int_{0}^{\tau} \int_{\mathbb{R}^{d}} \varrho_{\varepsilon}^{k-1}\left(\varrho * q_{\varepsilon}\right)^{2} d x d t\right)^{1 / 2} \\
& \quad+k\left(\int_{0}^{\tau} \int_{\mathbb{R}^{d}}\left|\nabla \varrho_{\varepsilon}\right|^{2} \varrho_{\varepsilon}^{k-1} d x d t\right)^{1 / 2}\left(\int_{0}^{\tau} \int_{\mathbb{R}^{d}} \varrho_{\varepsilon}^{k-1} \sum_{i=1}^{d}\left[\left(b^{i} \varrho\right) * w_{\varepsilon}\right]^{2} d x d t\right)^{1 / 2}
\end{aligned}
$$


where $q_{\varepsilon}$ is the same as in (2.10). By the inequality $a b \leq \alpha a^{2} / 4+b^{2} / \alpha$ we obtain the estimate

$$
\begin{aligned}
R_{\varepsilon} \leq-\frac{1}{2} k \alpha \int_{0}^{\tau} \int_{\mathbb{R}^{d}}\left|\nabla \varrho_{\varepsilon}\right|^{2} \varrho_{\varepsilon}^{k-1} d x d t+\frac{k d^{3} \lambda^{2}}{\alpha} \int_{0}^{\tau} \int_{\mathbb{R}^{d}} \varrho_{\varepsilon}^{k-1}\left(\varrho * q_{\varepsilon}\right)^{2} d x d t & \\
& +\frac{k}{\alpha} \int_{0}^{\tau} \int_{\mathbb{R}^{d}} \varrho_{\varepsilon}^{k-1} \sum_{i=1}^{d}\left[\left(b^{i} \varrho\right) * w_{\varepsilon}\right]^{2} d x d t .
\end{aligned}
$$

Combining our bounds on $L_{\varepsilon}$ and $R_{\varepsilon}$ we arrive at the inequality

$$
\begin{aligned}
& \frac{2}{\alpha k(k+1)} \int_{\mathbb{R}^{d}} \varrho_{\varepsilon}(\tau, x)^{k+1} d x+\int_{0}^{\tau} \int_{\mathbb{R}^{d}}\left|\nabla \varrho_{\varepsilon}\right|^{2} \varrho_{\varepsilon}^{k-1} d x d t \\
& \leq C(\alpha, \lambda, d)\left(\int_{0}^{\tau} \int_{\mathbb{R}^{d}} \varrho_{\varepsilon}^{k-1}\left(\varrho * q_{\varepsilon}\right)^{2} d x d t+\int_{0}^{\tau} \int_{\mathbb{R}^{d}} \varrho_{\varepsilon}^{k-1} \sum_{i=1}^{d}\left[\left(b^{i} \varrho\right) * w_{\varepsilon}\right]^{2} d x d t\right) \\
& \quad+\frac{2}{\alpha k(k+1)} \int_{\mathbb{R}^{d}} \varrho(0, x)^{k+1} d x .
\end{aligned}
$$

Note that $\left|\left(b^{i} \varrho\right) * w_{\varepsilon}\right|^{2} \leq \varrho_{\varepsilon}\left(\left|b^{i}\right|^{2} \varrho\right) * w_{\varepsilon}$. By Hölder's inequality we have

$$
\begin{gathered}
\int_{\mathbb{R}^{d}} \varrho_{\varepsilon}^{k-1}\left|\left(b^{i} \varrho\right) * w_{\varepsilon}\right|^{2}(t, x) d x \\
\leq \int_{\mathbb{R}^{d}} \varrho_{\varepsilon}^{k}\left(\left|b^{i}\right|^{2} \varrho\right) * w_{\varepsilon}(t, x) d x=\int_{\mathbb{R}^{d}}\left(\int_{\mathbb{R}^{d}} \varrho_{\varepsilon}(t, x)^{k} w_{\varepsilon}(x-y) d x\right)\left|b^{i}(t, y)\right|^{2} \varrho(t, y) d y \\
\leq\left\|\left(b^{i}(t, \cdot)\right)^{2}\right\|_{L^{s / 2}\left(\mu_{t}\right)}\left(\int_{\mathbb{R}^{d}}\left(\int_{\mathbb{R}^{d}} \varrho_{\varepsilon}(t, x)^{k} w_{\varepsilon}(x-y) d x\right)^{s /(s-2)} \varrho(t, y) d y\right)^{(s-2) / s} \\
\left.\leq\left\|b^{i}(t, \cdot)\right\|_{L^{s}\left(\mu_{t}\right)}^{2}\left(\int_{\mathbb{R}^{d}} \varrho_{\varepsilon}(t, x)^{k(s-2) / s} w_{\varepsilon}(x-y) d x\right) \varrho(t, y) d y\right)^{(s-2) / s} \\
=\left\|b^{i}(t, \cdot)\right\|_{L^{s}\left(\mu_{t}\right)}^{2}\left(\int_{\mathbb{R}^{d}} \varrho_{\varepsilon}(t, x)^{k s /(s-2)+1} d x\right)^{(s-2) / s} .
\end{gathered}
$$

Similarly, taking into account the estimate

$$
\left|\varrho * q_{\varepsilon}(t, x)\right|^{2} \leq \varrho_{\varepsilon}(t, x) \int_{\mathbb{R}^{d}} \varrho(t, y) \frac{|x-y|^{4}}{\varepsilon^{4}} w_{\varepsilon}(x-y) d y
$$

we obtain

$$
\begin{gathered}
\int_{\mathbb{R}^{d}} \varrho_{\varepsilon}^{k-1}\left(\varrho * q_{\varepsilon}\right)^{2}(t, x) d x \leq \int_{\mathbb{R}^{d}} \int_{\mathbb{R}^{d}} \varrho_{\varepsilon}(t, x)^{k} \varrho(t, y) \frac{|x-y|^{4}}{\varepsilon^{4}} w_{\varepsilon}(x-y) d x d y \\
\leq \gamma(s) \int_{\mathbb{R}^{d}} \varrho(t, y)\left(\int_{\mathbb{R}^{d}} \varrho_{\varepsilon}(t, x)^{k s /(s-2)} w_{\varepsilon}(x-y) d x\right)^{s /(s-2)} d y \\
\leq \gamma(s)\left(\int_{\mathbb{R}^{d}} \int_{\mathbb{R}^{d}} \varrho(t, y) \varrho_{\varepsilon}(t, x)^{k s /(s-2)} w_{\varepsilon}(x-y) d x d y\right)^{s /(s-2)} \\
\quad=\gamma(s)\left(\int_{\mathbb{R}^{d}} \varrho_{\varepsilon}(t, x)^{k s /(s-2)+1} d x\right)^{(s-2) / s}
\end{gathered}
$$

where

$$
\gamma(s):=\left(\int_{\mathbb{R}^{n}}|x|^{2 s} g(x) d x\right)^{2 / s}
$$

Set

$$
B(s):=\max _{t \in[0,1]}\|b(t, \cdot)\|_{L^{s}\left(\mu_{t}\right)}^{2} .
$$


Finally, we obtain

$$
\begin{aligned}
\frac{2}{\alpha k(k+1)} & \int_{\mathbb{R}^{d}} \varrho_{\varepsilon}(\tau, x)^{k+1} d x+\int_{0}^{\tau} \int_{\mathbb{R}^{d}}\left|\nabla \varrho_{\varepsilon}\right|^{2} \varrho_{\varepsilon}^{k-1} d x d t \\
& \leq C(\alpha, \lambda, d)(\gamma(s)+B(s)) \int_{0}^{\tau}\left(\int_{\mathbb{R}^{d}} \varrho_{\varepsilon}(t, x)^{k s /(s-2)+1} d x\right)^{(s-2) / s} d t \\
& \quad+\frac{2}{\alpha k(k+1)} \int_{\mathbb{R}^{d}} \varrho(0, x)^{k+1} d x .
\end{aligned}
$$

Passing to the limit as $\varepsilon \rightarrow 0$, we obtain the required estimate for almost every $\tau \in[0, T]$. Indeed, for almost every $\tau$ we have $\lim _{\varepsilon \rightarrow 0} \varrho_{\varepsilon}(\tau, x)=\varrho(\tau, x)$ for almost all $x$, and the righthand side of the above inequality remains bounded as $\varepsilon \rightarrow 0$. This yields that $\varrho(\tau, \cdot) \in$ $W_{l o c}^{1,1}$ for almost all $\tau$. Hence $\lim _{\varepsilon \rightarrow 0} \nabla \varrho_{\varepsilon}(\tau, x)=\nabla \varrho(\tau, x)$ for almost all $x$, which gives the indicated estimate by Fatou's theorem.

In the case of condition (3.3) the proof repeats entirely the foregoing reasoning except for the estimate of the integral of $\varrho_{\varepsilon}^{k-1}\left|\left(b^{i} \varrho\right) * w_{\varepsilon}\right|^{2}$, for which we have

$$
\begin{gathered}
\int_{0}^{\tau} \int_{\mathbb{R}^{d}} \varrho_{\varepsilon}^{k-1}\left|\left(b^{i} \varrho\right) * w_{\varepsilon}\right|^{2} d x d t \\
\leq \int_{0}^{\tau} \int_{\mathbb{R}^{d}} \varrho_{\varepsilon}^{k}\left(\left|b^{i}\right|^{2} \varrho\right) * w_{\varepsilon} d x d t=\int_{0}^{\tau} \int_{\mathbb{R}^{d}}\left(\int_{\mathbb{R}^{d}} \varrho_{\varepsilon}(t, x)^{k} w_{\varepsilon}(x-y) d x\right)\left|b^{i}(t, y)\right|^{2} \varrho(t, y) d y d t \\
\leq\left\|\left(b^{i}\right)^{2}\right\|_{L^{s / 2}(\mu)}\left(\int_{0}^{\tau} \int_{\mathbb{R}^{d}}\left(\int_{\mathbb{R}^{d}} \varrho_{\varepsilon}(t, x)^{k} w_{\varepsilon}(x-y) d x\right)^{s /(s-2)} \varrho(t, y) d y d t\right)^{(s-2) / s} \\
\leq\|b\|_{L^{s}(\mu)}^{2}\left(\int_{0}^{\tau} \int_{\mathbb{R}^{d}} \varrho_{\varepsilon}(t, x)^{k s /(s-2)+1} d x d t\right)^{(s-2) / s}
\end{gathered}
$$

by Hölder's inequality.

Remark 3.1. It is seen from the proof that the assumption that the integrals of $\varrho(t, x)$ with respect to $x$ equal 1 can be replaced by the assumption that these integrals are uniformly bounded.

Theorem 3.1. Suppose that under the hypotheses of Theorem 2.1] we have additionally

$$
\sup _{t \in[0,1]}\|b(t, \cdot)\|_{L^{d}\left(\mu_{t}\right)}<\infty
$$

and $\mu_{0}=\varrho(0, \cdot) d x$, where $\varrho(0, \cdot) \in L^{p}\left(\mathbb{R}^{d}\right)$ for all $p \in[1,+\infty)$. Then

$$
\varrho \in L^{p, q}\left([0, \tau] \times \mathbb{R}^{d}\right)
$$

for all $p, q \in[1,+\infty)$ and $\tau \in(0,1)$.

Proof. Let us consider the case $d>2$. Let $\varrho \in L^{k s /(s-2)+1, k+(s-2) / s}\left([0, \tau] \times \mathbb{R}^{d}\right)$, where $s>2$ and $k \geq 2 / s$. Then by Lemma 3.2 we obtain the estimate

$$
\begin{aligned}
& \int_{0}^{\tau} \int_{\mathbb{R}^{d}}|\nabla \varrho(t, x)|^{2} \varrho(t, x)^{k-1} d x d t \\
& \quad \leq C(\alpha, \lambda, d, s) \int_{0}^{\tau}\left(\int_{\mathbb{R}^{d}} \varrho(t, x)^{k s /(s-2)+1} d x\right)^{(s-2) / s} d t+\frac{2}{\alpha k(k+1)} \int_{\mathbb{R}^{d}} \varrho(0, x)^{k+1} d x .
\end{aligned}
$$

By the Sobolev embedding theorem applied to the functions $x \mapsto \varrho(t, x)^{(k+1) / 2}$ with

$$
\left|\nabla \varrho^{(k+1) / 2}\right|^{2}=\frac{(k+1)^{2}}{4}\left|\varrho^{(k-1) / 2} \nabla \varrho\right|^{2}
$$


we have

$$
\begin{aligned}
& \frac{4}{(k+1)^{2}} \int_{0}^{\tau}\left(\int_{\mathbb{R}^{d}} \varrho(t, x)^{d(k+1) /(d-2)} d x\right)^{(d-2) / d} d t \leq \int_{0}^{\tau} \int_{\mathbb{R}^{d}}|\nabla \varrho(t, x)|^{2} \varrho(t, x)^{k-1} d x d t \\
& \quad \leq \widetilde{C}(\alpha, \lambda, d, s) \int_{0}^{\tau}\left(\int_{\mathbb{R}^{d}} \varrho(t, x)^{k s /(s-2)+1} d x\right)^{(s-2) / s} d t+\frac{2}{\alpha k(k+1)} \int_{\mathbb{R}^{d}} \varrho(0, x)^{k+1} d x .
\end{aligned}
$$

Thus, one has

$$
\begin{aligned}
& \left(\|\varrho\|_{d(k+1) /(d-2), k+1, \tau}\right)^{k+1} \\
& \quad \leq C(\alpha, \lambda, d, s, k)\left(\|\varrho\|_{k s /(s-2)+1, k+(s-2) / s, \tau}\right)^{k+(s-2) / s}+M(k, \alpha),
\end{aligned}
$$

where

$$
M(k, \alpha):=\frac{k+1}{2 \alpha k} \int_{\mathbb{R}^{d}} \varrho(0, x)^{k+1} d x
$$

Now set

$$
p_{n}:=p_{n-1}+\frac{2}{d-2}, \quad q_{n}:=q_{n-1}+\frac{2}{d}, \quad q_{1}=1, \quad p_{1}=\frac{d}{d-2} .
$$

By Theorem 2.1 we have $\varrho \in L^{p_{1}, 1}\left([0, \tau] \times \mathbb{R}^{d}\right)$ for all $\tau<1$. This enables us to start iterations based on (3.6). Namely, if in (3.6) we set $s=d$ and $k=q_{n-1}-(d-2) / d=q_{n}-1$, then we arrive at the estimate

$$
\left(\|\varrho\|_{p_{n}, q_{n}, \tau}\right)^{q_{n}} \leq C\left(\alpha, \lambda, d, d, q_{n}-1\right)\left(\|\varrho\|_{p_{n-1}, q_{n-1}, \tau}\right)^{q_{n-1}}+M\left(q_{n}-1, \alpha\right) .
$$

Since $p_{n} \rightarrow \infty$ and $q_{n} \rightarrow \infty$ as $n \rightarrow \infty$, the theorem is proven in the case $d>2$. The cases $d=1$ and $d=2$ are even simpler, because in the Sobolev inequality in place of the exponent $d /(d-2)$ one can take any number $r>1$. However, we need not consider these cases separately and deduce them from the result for $d=3$. To this end, we pass from the function of two variables to the function of three variables $u=\varrho\left(t, x_{1}, x_{2}\right) g\left(x_{3}\right)$, where $g$ is the standard Gaussian density. The measure $u d x d t$ satisfies our equation on $[0,1) \times \mathbb{R}^{3}$ with the coefficients $a^{i j}$ and $b^{i}$ that coincide with the initial ones if $i, j \leq 2$, and $a^{33}=1, a^{3 j}=a^{j 3}=0, b^{3}(t, x)=-x_{3}$.

Theorem 3.2. Suppose that under the hypotheses of Theorem 2.1 for some $\beta>d+2$ we have $|b| \in L^{\beta}(\mu)$ and $\varrho(0, \cdot) \in L^{\infty}\left(\mathbb{R}^{d}\right)$. Supppose that either $\sup _{t \in[0,1]}\|b(t, \cdot)\|_{L^{d}\left(\mu_{t}\right)}<\infty$ or $\varrho \in L^{p}\left([0, \tau] \times \mathbb{R}^{d}\right)$, for all $\tau<1$ with some $p>1$. Then $\varrho \in L^{\infty}\left([0, \tau] \times \mathbb{R}^{d}\right)$ for every $\tau<1$.

Proof. Let $d>2$. Let us fix $\tau<1$. Let $\varrho \in L^{k \beta /(\beta-2)+1}\left([0, \tau] \times \mathbb{R}^{d}\right)$, where $k>0$. Then by (3.4) we obtain

$$
\begin{aligned}
& \int_{0}^{\tau} \int_{\mathbb{R}^{d}}|\nabla \varrho(t, x)|^{2} \varrho(t, x)^{k-1} d x d t \\
& \quad \leq C(\alpha, \lambda, d, \beta)\left(\int_{0}^{\tau} \int_{\mathbb{R}^{d}} \varrho(t, x)^{k \beta /(\beta-2)+1} d x d t\right)^{(\beta-2) / \beta}+\frac{2}{\alpha k(k+1)} \int_{\mathbb{R}^{d}} \varrho(0, x)^{k+1} d x .
\end{aligned}
$$

Therefore,

$$
\begin{aligned}
& \frac{4}{(k+1)^{2}}\left(\left\|\nabla\left(\varrho^{(k+1) / 2}\right)\right\|_{L^{2}\left([0, \tau] \times \mathbb{R}^{d}\right)}\right)^{2} \\
& \quad \leq C(\alpha, \lambda, d, \beta)\left(\int_{0}^{\tau} \int_{\mathbb{R}^{d}} \varrho(t, x)^{k \beta /(\beta-2)+1} d x d t\right)^{(\beta-2) / \beta}+\frac{2}{\alpha k(k+1)} \int_{\mathbb{R}^{d}} \varrho(0, x)^{k+1} d x .
\end{aligned}
$$


By inequality 3.4 we have for almost all $t<\tau$

$$
\begin{aligned}
\int_{\mathbb{R}^{d}} \varrho(t, x)^{k+1} d x & \\
& \leq k(k+1) C(\alpha, \lambda, d, \beta)\left(\int_{0}^{\tau} \int_{\mathbb{R}^{d}} \varrho^{k \beta /(\beta-2)+1} d x d t\right)^{(\beta-2) / \beta}+\int_{\mathbb{R}^{d}} \varrho(0, x)^{k+1} d x,
\end{aligned}
$$

whence we obtain

$$
\begin{aligned}
& \left(\left\|\varrho^{(k+1) / 2}\right\|_{2, \infty, \tau}\right)^{2} \\
& \quad \leq k(k+1) C(\alpha, \lambda, d, \beta)\left(\int_{0}^{\tau} \int_{\mathbb{R}^{d}} \varrho^{k \beta /(\beta-2)+1} d x d t\right)^{(\beta-2) / \beta}+\int_{\mathbb{R}^{d}} \varrho(0, x)^{k+1} d x .
\end{aligned}
$$

Lemma 3.1 for $u=\varrho^{(k+1) / 2}$ and $p=q=2(d+2) / d$ yields the estimate

$$
\left\|\varrho^{(k+1) / 2}\right\|_{p, q, \tau} \leq C(d, 2(d+2) / d)\left(\left\|\nabla\left(\varrho^{(k+1) / 2}\right)\right\|_{L^{2}\left([0, \tau] \times \mathbb{R}^{d}\right)}+\left\|\varrho^{(k+1) / 2}\right\|_{2, \infty, \tau}\right) .
$$

By using the inequality $(a+b)^{2} \leq 2 a^{2}+2 b^{2}$ and the bounds found above we obtain

$$
\begin{aligned}
& \left(\|\varrho\|_{L^{(d+2)(k+1) / d\left([0, \tau] \times \mathbb{R}^{d}\right)}}\right)^{k+1} \leq 4(k+1)^{2} C(\alpha, \lambda, d, \beta)\left(\|\varrho\|_{L^{k \beta /(\beta-2)+1}\left([0, \tau] \times \mathbb{R}^{d}\right)}\right)^{k+(\beta-2) / \beta} \\
& +\left(2+\frac{k+1}{\alpha k}\right) \int_{\mathbb{R}^{d}} \varrho(0, x)^{k+1} d x .
\end{aligned}
$$

Since $\varrho(0, \cdot) \in L^{\infty}\left([0, \tau] \times \mathbb{R}^{d}\right)$, there exists a constant $C$ such that

$$
\left(2+\frac{k+1}{\alpha k}\right) \int_{\mathbb{R}^{d}} \varrho(0, x)^{k+1} d x \leq C^{k+1} .
$$

Now we set

$$
\begin{aligned}
& p_{n}=\frac{(d+2)(\beta-2)}{d \beta}\left(p_{n-1}+\frac{2}{\beta-2}\right), \quad p_{1}=\frac{d}{d-2}, \\
& A_{n}=\|\varrho\|_{L^{p_{n}\left([0, \tau] \times \mathbb{R}^{d}\right)}}, \quad C_{1}=4\left(\frac{d}{d+2}\right)^{2} C(\alpha, \lambda, d, \beta) .
\end{aligned}
$$

We have $\varrho \in L^{p_{1}}\left([0, \tau] \times \mathbb{R}^{d}\right)$ by Theorem 3.1] if $\sup _{t}\|b(t, \cdot)\|_{L^{d}\left(\mu_{t}\right)}<\infty$; if $\varrho \in L^{p} p>1$, then the same can be readily deduced from (3.7). Note that

$$
1<\frac{(d+2)(\beta-2)}{d \beta} \leq \frac{d}{d-2}
$$

since $\beta>d+2$. Hence $p_{n} \geq((d+2)(\beta-2) /(d \beta))^{n}$. In order to prove the membership of $\varrho$ in the space $L^{\infty}\left([0, \tau] \times \mathbb{R}^{d}\right)$, it suffices to establish the uniform boundedness of $\left\{A_{n}\right\}$. Suppose that $A_{n} \rightarrow \infty$. Then there exists $N$ such that for every $n>N$ we have

$$
\frac{C}{A_{n-1}}<1, \quad\left(A_{n-1}\right)^{-2 / \beta}<1, \quad\left(\frac{C}{A_{n-1}}\right)^{p_{n} d /(d+2)}<1 .
$$

Let $k:=p_{n} d /(d+2)-1$. Then

$$
p_{n-1}=\frac{k \beta}{\beta-2}+1, \quad p_{n-1} \frac{\beta-2}{\beta}-p_{n} \frac{d}{d+2}=-\frac{2}{\beta}
$$

so (3.7) yields

$$
\left(A_{n}\right)^{p_{n} d /(d+2)} \leq p_{n}^{2} C_{1}\left(A_{n-1}\right)^{p_{n-1}(\beta-2) / \beta}+C^{p_{n} d /(d+2)} .
$$

Therefore,

$$
\left(\frac{A_{n}}{A_{n-1}}\right)^{p_{n} d /(d+2)} \leq p_{n}^{2} C_{1}\left(A_{n-1}\right)^{-2 / \beta}+\left(\frac{C}{A_{n-1}}\right)^{p_{n} d /(d+2)} \leq p_{n}^{2} C_{1}+1 .
$$

whence we obtain

$$
\ln A_{n}-\ln A_{n-1} \leq \frac{d+2}{p_{n} d} \ln \left(p_{n}^{2} C_{1}+1\right), \quad n>N
$$


Since $p_{n} \geq((d+2)(\beta-2) /(d \beta))^{n}$, the above estimate yields convergence of the series of $\ln A_{n}-\ln A_{n-1}$, which contradicts the unboundedness of $A_{n}$. The case $d \leq 2$ is justified in the same manner as in the previous theorem.

Remark 3.2. (i) According to Remark 2.1, no integrability $\varrho(0, x) \ln \varrho(0, x)$ in Theorems 3.1 and 3.2 is required, since the integrability of $\varrho(0, x) \max (0, \ln \varrho(0, x))$ follows by the inclusion $\varrho(0, \cdot) \in L^{p}\left(\mathbb{R}^{d}\right)$ with $p>1$.

(ii) It is seen from the proof and Remark 3.1 that the assumption in Theorem 3.1 and Theorem 3.2 that the integrals of $\varrho(t, x)$ with respect to $x$ equal 1 can be replaced by the assumption that these integrals are uniformly bounded.

(iii) Let us note that if in Theorem 3.2 it is given in advance that $\varrho \in L^{p}\left([0, \tau] \times \mathbb{R}^{d}\right)$ for some $p>1$, then we need not require the integrability of the function $|\ln (1+|x|)|^{2} \varrho(t, x)$, but the boundedness of $\varrho(0, x)$ is important.

Now we employ the proven theorem for obtaining upper bounds on $\varrho$. As in the elliptic case considered in the papers [14, [5], 6], the idea is this: in order to obtain a pointwise estimate $\varrho(t, x) \leq \Phi(t, x)^{-1}$, one has to consider the measure $\nu:=\Phi \cdot \mu$ and establish the boundedness of its density. We shall consider functions $\Phi$ that do not depend on $t$. If $\Phi$ has locally bounded first and second order derivatives, then the measure $\nu$ satisfies the equation

$$
L^{*} \nu=\left(a^{i j} \partial_{x_{i}} \partial_{x_{j}} \Phi\right) \varrho+2 \partial_{x_{i}} \Phi \partial_{x_{j}}\left(a^{i j} \varrho\right)-b^{i} \partial_{x_{i}} \Phi \varrho=-L \Phi \cdot \varrho+2 \partial_{x_{j}}\left(a^{i j} \partial_{x_{i}} \Phi \varrho\right)
$$

understood in the same sense as (1.1).

Theorem 3.3. Suppose that all hypotheses of Theorem 3.2 are fulfilled and we are given a function $\Phi \geq c>0$ on $\mathbb{R}^{d}$ with locally bounded second order derivatives such that $\varrho(0, x) \leq C \Phi(x)^{-1}, \Phi \in L^{1}\left(\mu_{0}\right)$ and

$$
\Phi^{1+\varepsilon},|L \Phi|^{\beta / 2} \Phi^{1-\beta / 2},|A \nabla \Phi|^{\beta} \Phi^{1-\beta} \in L^{1}(\mu), \sup _{t \in[0,1]} \int_{\mathbb{R}^{d}} \Phi(x) \varrho(t, x) d x<\infty
$$

with some $\varepsilon>0$. Then for every $\tau<1$ there is a number $C_{\tau}$ such that

$$
\varrho(t, x) \leq C_{\tau} \Phi(x)^{-1} \quad \text { for almost all }(t, x) \in[0, \tau) .
$$

Proof. It is seen from the reasoning used in the proof of Theorem 3.2 and Remark 3.2 that it suffices to establish estimate (3.4) with $s=\beta$ for the measure $\nu=\Phi \cdot \mu$ whose density belongs to $L^{1+\varepsilon}\left([0,1] \times \mathbb{R}^{d}\right)$ by the boundedness of $\varrho$. In that estimate a homogeneous equation was concerned, and the measures $\mu_{t}$ were probabilities. However, under present assumptions the same estimate remains valid in the presence of the indicated right-hand side as well if in place of the condition $\mu_{t}\left(\mathbb{R}^{d}\right)=1$ we assume only the uniform boundedness of measures $\mu_{t}$. Indeed, on the right-hand side of (3.5) with $\nu$ in place of $\mu$, i.e., with $u:=\Phi \varrho$ in place of $\varrho$, there appears additionally the integral of the expression

$$
-(L \Phi \cdot \varrho) * w_{\varepsilon}\left[u * w_{\varepsilon}\right]^{k}+\left[2 \partial_{x_{j}}\left(a^{i j} \partial_{x_{i}} \Phi \varrho\right)\right] * w_{\varepsilon}\left[u * w_{\varepsilon}\right]^{k} .
$$

Let us set $\xi:=L \Phi / \Phi, \eta:=|A \nabla \Phi| / \Phi$ and estimate this integral $J$ as follows:

$$
\begin{gathered}
J=-\int_{0}^{\tau} \int_{\mathbb{R}^{d}}(\xi u)_{\varepsilon} u_{\varepsilon}^{k} d x d t-2 k \int_{0}^{\tau} \int_{\mathbb{R}^{d}}\left(a^{i j} \partial_{x_{i}} \Phi \varrho\right)_{\varepsilon} u_{\varepsilon}^{k-1} \partial_{x_{j}} u_{\varepsilon} d x d t \\
\leq\left(\int_{0}^{\tau} \int_{\mathbb{R}^{d}}|\xi|^{\beta / 2} u d x d t\right)^{2 / \beta}\left(\int_{0}^{\tau} \int_{\mathbb{R}^{d}} u_{\varepsilon}^{k \beta /(\beta-2)+1} d x d t\right)^{(\beta-2) / \beta} \\
+2 k\left(\int_{0}^{\tau} \int_{\mathbb{R}^{d}}(\eta u)_{\varepsilon}^{2} u_{\varepsilon}^{k-1} d x d t\right)^{1 / 2}\left(\int_{0}^{\tau} \int_{\mathbb{R}^{d}}\left|\nabla u_{\varepsilon}\right|^{2} u_{\varepsilon}^{k-1} d x d t\right)^{1 / 2} .
\end{gathered}
$$

It remains to observe that there hold the equality

$$
\int_{0}^{\tau} \int_{\mathbb{R}^{d}}|\xi|^{\beta / 2} u d x d t=\int_{0}^{\tau} \int_{\mathbb{R}^{d}}|L \Phi|^{\beta / 2} \Phi^{1-\beta / 2} \varrho d x d t
$$


and the inequality

$$
\int_{0}^{\tau} \int_{\mathbb{R}^{d}}(\eta u)_{\varepsilon}^{2} u_{\varepsilon}^{k-1} d x d t \leq\left(\int_{0}^{\tau} \int_{\mathbb{R}^{d}} \eta^{\beta} \Phi \varrho d x d t\right)^{2 / \beta}\left(\int_{0}^{\tau} \int_{\mathbb{R}^{d}} u_{\varepsilon}^{(k \beta) /(\beta-2)+1} d x d t\right)^{(\beta-2) / \beta},
$$

which is verified in the same manner as in Lemma 3.2. Since $s=\beta$, one has $r^{\prime} \leq \beta / 2$.

Example 3.1. Suppose that $A$ and $A^{-1}$ are uniformly bounded, $A$ is uniformly Lipschitzian in $x$, and for some $\beta>d+2, r>0, \varepsilon>0, K>0$ one has

$$
|b| \in L^{\beta}(\mu), \exp \left[(2 K+\varepsilon)|x|^{r}\right] \in L^{1}(\mu), \sup _{t \in[0,1]} \int_{\mathbb{R}^{d}} \exp \left(K|x|^{r}\right) \varrho(t, x) d x<\infty .
$$

Let $\sup _{t \in[0,1]}\|b(t, \cdot)\|_{L^{d}\left(\mu_{t}\right)}<\infty$. Finally, let the function $\exp \left(K|x|^{r}\right) \varrho(0, x)$ be bounded and integrable on $\mathbb{R}^{d}$. Then for every $\tau<1$ there is a number $C(\tau)$ such that

$$
\varrho(t, x) \leq C(\tau) \exp \left(-K|x|^{r}\right), \quad(t, x) \in[0, \tau] \times \mathbb{R}^{d} .
$$

In order to ensure condition (3.8) and the assumptions on $b$ and $\varrho(0, \cdot)$ it suffices to have the estimates $|b(t, x)| \leq C \exp \left(2 K \beta^{-1}|x|^{r}\right), \varrho(0, x) \leq C \exp \left(-K^{\prime}|x|^{r}\right)$ with $K^{\prime}>K$ and

$$
(x, b(t, x)) \leq c_{1}-c_{2}|x|^{r}, c_{2}>2 r K \sup _{t, x}\|A(t, x)\| .
$$

Indeed, let $\Phi \in C^{2}\left(\mathbb{R}^{d}\right), \Phi(x)=\exp \left(K|x|^{r}\right) \quad|x| \geq 1$. All hypotheses of Theorem 3.3 are fulfilled. Under condition (3.9) we pick $\delta \in(0, \varepsilon)$ such that one has the inequality $r(2 K+$ $\delta) \sup _{t, x}\|A(t, x)\|<c_{2}$, and take a function $V \in C^{2}\left(\mathbb{R}^{d}\right)$ that equals $\exp \left[(2 K+\delta)|x|^{r}\right]$ if $|x| \geq 1$. Then, for some $c$, we have the estimate $L V \leq c$. It follows from [1] that a solution exists and the norms $\|V \varrho(t, \cdot)\|_{L^{1}\left(\mathbb{R}^{d}\right)}$ are uniformly bounded. Other assumptions of Theorem 3.3 are fulfilled as well. Similarly, under weaker conditions, one can obtain a power bound.

Analogous theorems are valid in the situation of the second theorem of the previous section.

Most of the work has been done during visits of the first and third authors to the University of Bielefeld.

\section{REFERENCES}

[1] Bogachev V.I., Da Prato G., Röckner M. Existence of solutions to weak parabolic equations for measures. - Proc. London Math. Soc., 2004, v. 88, no. 3, p. 753-774.

[2] Bogachev V.I., Da Prato G., Röckner M. On solvability of weak parabolic equations for measures. Preprint BiBoS, Bielefeld University, 2005.

[3] Bogachev V.I., Krylov N.V., Röckner M. On regularity of transition probabilities and invariant measures of singular diffusions under minimal conditions. - Comm. Partial Diff. Equations, 2001, v. 26, no. 11-12, p. 2037-2080.

[4] Bogachev V.I., Krylov N.V., Röckner M. Regularity of invariant measures: the case of non-constant diffusion part. - J. Funct. Anal., 1996, v. 138, no. 1, p. 223-242.

[5] Bogachev V.I., Krylov N.V., Röckner M. Regularity and global bounds for densities of invariant measures of diffusion processes. - Dokl. Russian Acad. Sci. 2005. V. 405, N 5 (in Russian); English. transl.: Dokl. Math. 2005.

[6] Bogachev V.I., Krylov N.V., Röckner M. Elliptic equations for measures: regularity and global bounds of densities. - BiBoS Preprint 05-06-186, Bielefeld University; to appear in J. Math. Pures Appl.

[7] Bogachev V.I., Röckner M. Regularity of invariant measures on finite and infinite dimensional spaces and applications. - J. Funct. Anal., 1995, v. 133, no. 1, p. 168-223.

[8] Bogachev V.I., Röckner M., Wang F.-Y. Elliptic equations for invariant measures on finite and infinite dimensional manifolds. - J. Math. Pures Appl., 2001, v. 80, p. 177-221.

[9] Carlen E.A. Conservative diffusions. - Comm. Math. Phys., 1984, v. 94, no. 3, p. 293-315.

[10] Cattiaux P., Fradon M. Entropy, reversible diffusion processes, and Markov uniqueness. - J. Funct. Anal., 1996, v. 138, no. 1, p. 243-272.

[11] Cattiaux P., Léonard C. Minimization of the Kullback information of diffusion processes. - Ann. Inst. H. Poincaré, 1994, v. 30, no. 1, p. 83-132; correction: ibid., 1995, v. 31, no. 4, p. 705-707.

[12] Föllmer H. Random fields and diffusion processes. - Lect. Notes in Math., 1980, v. 1362, p. 101-203. 
[13] Ladyz'enskaya O.A., Solonnikov V.A., Ural'tseva N.N. Linear and quasilinear equations of parabolic type. Amer. Math. Soc., Rhode Island, 1968.

[14] Metafune G., Pallara D., Rhandi A. Global regularity of invariant measures. - J. Funct. Anal., 2005, v. 223, p. 396-424.

[15] Nelson E. Quantum fluctuations. Princeton University Press, Princeton, 1984.

[16] Stannat W. Time-dependent diffusion operators on $L^{1}$. - Preprint SFB 343, Univ. Bielefeld, N 00080 (2000), $41 \mathrm{pp}$.

Department of Mechanics and Mathematics, Moscow State University, 119992 Moscow, Russia

Department of Mathematics, Purdue University, 150 N. University Str., West LafayETTE, IN 47907-2067, USA

Department of Mechanics and Mathematics, Moscow State University, 119992 Moscow, RUSSIA 